\title{
Syntaphilin-Mediated Docking of Mitochondria at the Growth Cone Is Dispensable for Axon Elongation In Vivo
}

\author{
Tine Verreet, ${ }^{1}$ CCory J. Weaver, ${ }^{1}$ Hiromu Hino, ${ }^{2}{ }^{\circledR}$ Masahiko Hibi, ${ }^{2}$ and ${ }^{10}$ Fabienne E. Poulain ${ }^{1}$
}

https://doi.org/10.1523/ENEURO.0026-19.2019

${ }^{1}$ Department of Biological Sciences, University of South Carolina, Columbia, South Carolina 29208, and ${ }^{2}$ Laboratory of Organogenesis and Organ Function, Bioscience and Biotechnology Center, Nagoya University, Nagoya 464-8601, Aichi, Japan

\begin{abstract}
Mitochondria are abundantly detected at the growth cone, the dynamic distal tip of developing axons that directs growth and guidance. It is, however, poorly understood how mitochondrial dynamics relate to growth cone behavior in vivo, and which mechanisms are responsible for anchoring mitochondria at the growth cone during axon pathfinding. Here, we show that in retinal axons elongating along the optic tract in zebrafish, mitochondria accumulate in the central area of the growth cone and are occasionally observed in filopodia extending from the growth cone periphery. Mitochondrial behavior at the growth cone in vivo is dynamic, with mitochondrial positioning and anterograde transport strongly correlating with growth cone behavior and axon outgrowth. Using novel zebrafish mutant lines that lack the mitochondrial anchoring proteins Syntaphilin a and b, we further show that Syntaphilins contribute to mitochondrial immobilization at the growth cone. Syntaphilins are, however, not required for proper growth cone morphology and axon growth in vivo, indicating that Syntaphilin-mediated anchoring of mitochondria at the growth cone plays only a minor role in elongating axons.
\end{abstract}

Key words: axon growth; confocal live imaging; mitochondria; Syntaphilin; visual system; zebrafish

\section{Significance Statement}

Proper axon elongation and pathfinding are essential for nervous system wiring. The growth cone, a dynamic structure at the distal end of axons, mediates axonal growth and guidance. Here, we describe for the first time in vivo the behavior of mitochondria at the growth cone of elongating axons. We show that mitochondria accumulate in the growth cone central area and are also present in its periphery. We further provide evidence that Syntaphilin, which immobilizes mitochondria along mature axons, also docks mitochondria at the growth cone. However, the loss of Syntaphilin did not cause a complete depletion of mitochondria from the growth cone and did not affect axon elongation, indicating that other mitochondriadocking factors regulate axon growth during development.

\section{Introduction}

Nervous system formation and function critically rely on mitochondria. The ability of mitochondria to produce ATP via oxidative phosphorylation and to buffer cytosolic cal-

Received January 19, 2019; accepted August 22, 2019; First published September 3, 2019.

The authors declare no competing financial interests. cium is especially important in neurons that have a high energy demand and require proper ion homeostasis. Mitochondrial dynamics, including transport, fission, and 
fusion, contribute to the correct distribution of mitochondria in axons and are therefore essential regulators of mitochondrial functions (Trevisan et al., 2018). Mitochondria are targeted to regions distant from the cell body, such as the axonal growth cone and synaptic terminals, through their active transport along microtubules (Melkov and Abdu, 2018). Overall, the importance of mitochondrial dynamics for proper neuronal development and function is emphasized by the large number of neurologic disorders caused by mutations affecting mitochondrial proteins (Misko et al., 2010; Bertholet et al., 2016).

During development, distal growing axons and growth cones contain higher densities of mitochondria compared with proximal axonal regions (Morris and Hollenbeck, 1993). This asymmetrical distribution is thought to be necessary for axon growth (Smith and Gallo, 2018). For instance, in vitro, increasing mitochondrial density in the distal axon and growth cone by overexpressing the mitochondrial biogenesis regulator peroxisome proliferatoractivated receptor gamma coactivator- $1 \alpha$ (PGC-1 $\alpha$ ) was shown to increase axonal length (Vaarmann et al., 2016). Other studies have reported that factors promoting or inhibiting axon growth regulate mitochondrial localization at the growth cone (Beck et al., 2012; Sainath et al., 2017). For example, adding the growth-promoting factor BDNF to cultured neurons increases distal mitochondrial density, whereas adding the repulsive guidance cue ephrin-A5 or using chondroitin sulfate proteoglycans as a nonpermissive substrate both cause mitochondria to leave the growth cone (Beck et al., 2012; Sainath et al., 2017). While these in vitro studies have highlighted a significant role for mitochondria in axon extension and growth cone morphology, mitochondrial dynamics at the growth cone during axon pathfinding have never been assessed in vivo.

The mechanisms responsible for maintaining mitochondria in distal growing axons are not yet fully understood. Mitochondria are able to attach to both microtubule and actin cytoskeletons (Boldogh and Pon, 2006), and could therefore be immobilized on microtubules in the growth

This work was supported by the National Institutes of Health (Grants R00NS-083714 and R01-NS-109197 to F.E.P.), the University of South Carolina SmartState Center for Childhood Neurotherapeutics (to F.E.P.), the University of South Carolina (Aspire I Grant to T.V.), and the Belgian American Educational Foundation (postdoctoral fellowship to T.V.).

T. Verreet's present address: VIB-KU Leuven Center for Brain \& Disease Research, Department of Neurosciences, 3000 Leuven, Belgium.

M. Hibi's present address: Division of Biological Science, Graduate School of Science, Nagoya University, Furo-cho, Chikusa-ku, Nagoya 464-8602, Japan.

Acknowledgments: We thank Bradley Brodie for performing in situ hybridization experiments, and Brian Wheeler for technical assistance and fish husbandry. We also thank Drs. Amar N. Kar and Jeffery L. Twiss for their help with RT-ddPCR experiments. Finally, we thank Olivia Spead, Amar N. Kar, and Jeffery $L$. Twiss for helpful comments while drafting this manuscript.

Correspondence should be addressed to Fabienne E. Poulain at Fpoulain@ mailbox.sc.edu.

https://doi.org/10.1523/ENEURO.0026-19.2019

Copyright (C) 2019 Verreet et al.

This is an open-access article distributed under the terms of the Creative Commons Attribution 4.0 International license, which permits unrestricted use, distribution and reproduction in any medium provided that the original work is properly attributed. cone central area after being transported along developing axons. Such stalling might be mediated by Syntaphilin (Snph), a mitochondrial membrane protein able to directly tether mitochondria to microtubules, thereby stalling mitochondrial transport (Kang et al., 2008). By immobilizing mitochondria, Snph was shown to reduce synaptic plasticity (Kang et al., 2008) and modulate axon branching (Courchet et al., 2013). Snph was further found to prevent mitochondrial transport toward the distal axon and inhibit axon regeneration after injury (Zhou et al., 2016). However, whether Snph also docks mitochondria at the growth cone during axon elongation has never been tested.

The zebrafish embryo offers a unique accessibility and transparency to monitor mitochondrial distribution and transport along axons in vivo (Plucińska et al., 2012; Campbell et al., 2014; Drerup et al., 2017; Wehnekamp et al., 2019). Here, we used time-lapse imaging of mitochondria in single retinal axons as they elongate along the optic tract in zebrafish to characterize for the first the time in vivo mitochondrial dynamics at the growth cone during axon pathfinding. We show that mitochondrial distribution at the growth cone correlates with axon growth status, and that mitochondrial transport toward the growth cone correlates significantly with axon elongation. We further demonstrate that Snph contributes to mitochondrial docking at the growth cone during axon pathfinding. However, growth cone morphology and axon elongation are unaffected in snph mutants, indicating that the direct anchoring of mitochondria to growth cone microtubules by Snph only plays a minor role in axon elongation.

\section{Materials and Methods}

\section{Zebrafish husbandry}

All animal procedures were performed in accordance with the University of South Carolina's Institutional Animal Care and Use Committee (IACUC). Zebrafish (Danio rerio) wild-type (WT; AB strain) and lakritz (lak) mutant (Kay et al., 2001) embryos were obtained from natural matings, raised at $28.5^{\circ} \mathrm{C}$ in $\mathrm{E} 3$ medium $(5 \mathrm{~mm} \mathrm{NaCl}, 0.17 \mathrm{~mm} \mathrm{KCl}$, $0.33 \mathrm{~mm} \mathrm{CaCl}_{2}$, and $0.33 \mathrm{~mm} \mathrm{MgSO}_{4}$ ) in the presence of $150 \mathrm{~mm}$ 1-phenyl-2-thiourea (PTU; Sigma-Aldrich) to prevent pigment formation, and staged by age and morphology (Kimmel et al., 1995). Zebrafish possess a polygenic sex determination system, and sex-associated chromosomal regions are not fixed for the species (Liew et al., 2012). Our experiments were conducted on embryos before the onset of sexual differentiation, which occurs only at $\sim 2.5$ months after metamorphosis is complete (Maack and Segner, 2003).

\section{TALEN-mediated mutagenesis and genotyping of Snph mutants}

The transcription activator-like effector nucleases (TALENs) to target zebrafish snpha and snphb were designed using TAL Effector Nucleotide Targeter 2.0 (https://tale-nt.cac.cornell.edu/node/add/talen; Cermak et al., 2011). The TALENs for snpha contained the following repeat variable di-residues (RVDs): HD NI HD NN HD HD NI HD NG NN HD NI NN HD NI NN and NI NN HD HD 
Table 1: Primers and probes used for ddPCR

\begin{tabular}{|c|c|c|c|c|}
\hline Gene & Forward primer & Reverse primer & TaqMan probe & $\begin{array}{l}\text { Amp } \\
117\end{array}$ \\
\hline snpha & GCAGCAGTTACTCAGCATCA & TGCCATGATTCTCACCACAG & TCCTGCAAGTGCACAGAGAGCATT & 117 \\
\hline snphb & CACCTGTCAGTAACCGTGAT & TATGTGACGCCTATGGGTTG & AGCAGCAGTAGCAATTCAGGGTCA & 107 \\
\hline gapdh & CCAAGGCTGTAGGCAAAGTA & GACTGTCAGATCCACAACAGAG & ACACGGAAGGCCATACCAGTAAGC & 101 \\
\hline $18 s$ & GCCGCTAGAGGTGAAATTCT & TCGGAACTACGACGGTATCT & CAAGACGGACGAAAGCGAAAGCAT & 129 \\
\hline
\end{tabular}

NN NG NN HD NI HD NN NG NI NN NI NG NN HD, which targeted the sequences CACGCCACTGCAGCAG and AGCCGTGCACGTAGATGC, respectively. The TALENs for snphb contained the following RVDs: NN NI NN HD NI NI NG NI HD HD NG HD NI HD HD HD and NN HD HD NG NN NI NG NN HD NI HD NI HD HD NG HD, which targeted GAGCAATACCTCACCC and GCCTGATGCACACCTC, respectively. The TALEN cDNAs were assembled as described previously (Sakuma et al., 2013) and subcloned into pCS2pTAL3DD and pCS2pTALRR (Dahlem et al., 2012). Capped RNAs were synthesized from Notldigested TALEN expression plasmids using SP6 RNA polymerase (Promega) in the presence of $\mathrm{m}^{\prime} \mathrm{G}\left(5^{\prime}\right) \mathrm{ppp}\left(5^{\prime}\right) \mathrm{G}$ RNA Cap Structure Analog (NEB). One nanoliter of solution containing a pair of TALEN RNAs $(0.4 \mu \mathrm{g} / \mu \mathrm{l}$ each) was injected into zebrafish embryos at the one-cell stage. Deletion mutations in the target region were detected using heteroduplex mobility analysis (Ota et al., 2013). The following primers were used: AGAATCATGGCATTCGTCCTC and TGAAGCCTCTCCACATTTTCTT to detect the 14 bp deletion in snpha; and AATGATAACCATGGCATTCGAC and CTITAAGCCGTGCTCTCAGGT to detect the 4 bp deletion in snphb. PCR products were separated on $12 \%$ or $20 \%$ Tris-borate-EDTA acrylamide gels or on a $4 \%$ Metaphor gel (Lonza). The snphb mutation was additionally detected using high-resolution melting analysis (Parant et al., 2009) using the following primers: AGCAATACCTCACTCCACTG and GCCTGATGCACACCTCTTTC.

\section{Cloning of snpha and snphb coding sequences}

mRNA from embryos at $24 \mathrm{~h}$ postfertilization (hpf) was isolated using TRIzol (Thermo Fisher Scientific) and the RNeasy Mini Kit (Qiagen). cDNA was prepared from RNA using SuperScript III First-Strand Synthesis System (Thermo Fisher Scientific). The following primers were used to amplify zebrafish snpha and snphb cDNA: snpha forward, TGTCCTTCTGCATCCATGTC; snpha reverse, TCAGATAGGTGTCGCTCTTTC; snphb forward, ATG TCTTCGCCTTCAAATAAAAG; and snphb reverse, TCATATATTCATTCCCCCTGG. Amplicons were subcloned into Invitrogen PCRII-TOPO (Thermo Fisher Scientific), and were sequenced to verify gene identity and confirm sequence orientation for the generation of sense and antisense RNA probes.

\section{DNA plasmid constructs}

All expression vectors were constructed using the Tol2kit Gateway cloning system (Kwan et al., 2007). We generated a pME-mitoEGFP entry clone by adding the mitochondrial targeting signal of the zebrafish cox8a gene to the $5^{\prime}$ end of EGFP sequence using a BP-compatible forward primer. We generated a pME-Lifeact-TagRFP en- try clone by fusing the Lifeact sequence (Riedl et al., 2008) upstream of TagRFP using a BP-compatible forward primer. Lifeact-TagRFP was used to label F-actin in retinal axons and improve the visualization of growth cone filopodia. We generated a pME-TagBFP-snphb by adding the coding sequences of TagBFP (Evrogen) and a linker peptide (SGLRSRV) to the $5^{\prime}$ end of snphb. The p3E-2ATagRFPCAAX-pA entry clone that encodes a $2 A$ peptide (Provost et al., 2007) and TagRFP targeted to the plasma membrane by the prenylation motif of Ras (Moriyoshi et al., 1996) was a gift from Dr. Kristen Kwan (University of Utah, Salt Lake City, UT). The p5E-is/2b plasmid that drives specific expression in retinal ganglion cells (RGCs) was described previously (Pittman et al., 2008). Final isl2b:mitoEGFP-2A-TagRFPCAAX, isl2b:Lifeact-TagRFP, and isl2b:TagBFP-snphb plasmids were generated using $\mathrm{LR}$ reactions with the pDestTol2pA2 backbone (Kwan et al., 2007).

\section{RNA isolation and reverse transcriptase-coupled droplet digital PCR}

For RNA isolation from whole embryos, 15 dechorionated WT embryos were lysed and homogenized in $500 \mu \mathrm{l}$ TRIzol at cleavage, pharyngula prim-5 (24 hpf), long-pec (48 hpf), protruding mouth (72 hpf), larval day 4 (96 hpf), and larval day 5 (120 hpf). For RNA isolation from eyes at 72 hpf, 50 eyes were manually dissected from WT or lak mutant embryos. Total RNA was isolated using the Directzol RNA Miniprep Kit (Zymo Research) and eluted into 20 $\mu l$ of nuclease-free water. cDNA was synthesized with 5 $\mu \mathrm{g}$ of purified RNA as an input using the SuperScript III System. Digital droplet PCR (ddPCR) was then performed on a QX200 AutoDG Instrument (Bio-Rad) using predesigned TaqMan primers and probes for zebrafish snpha, sphnb, gapdh, and 18s (Table 1). Briefly, ddPCR reactions were assembled using Bio-Rad $2 \times$ ddPCR Supermix for Probes (no dUTP) and contained $1 \mu \mathrm{g}$ of cDNA and 250 $\mathrm{nm}$ primers/probe. PCR amplification was performed with the following parameters: 39 cycles of $94^{\circ} \mathrm{C}$ for $30 \mathrm{~s}$ and $60^{\circ} \mathrm{C}$ for $1 \mathrm{~min}$.

\section{In situ hybridization}

In vitro transcription of digoxigenin-labeled probes was performed using the RNA Labeling Kit (Roche Diagnostics) according to manufacturer instructions. Embryos were dechorionated at the appropriate developmental stages and fixed in 4\% paraformaldehyde in PBS, $\mathrm{pH} 7.4$, for $2 \mathrm{~h}$ at room temperature and overnight at $4^{\circ} \mathrm{C}$. Wholemount in situ hybridization (ISH) was performed as described previously (Thisse and Thisse, 2008). Sense probes were used as controls. After staining, embryos were cleared in $80 \%$ glycerol. Images were acquired using an Olympus SZX16 stereomicroscope equipped with 
an Olympus DP80 dual color camera and Cellsens standard software.

\section{Imaging of mitochondria in retinal axons}

Both isl2b:mitoEGFP-2A-TagRFPCAAX (30 pg) and isl2b:LifeAct-TagRFP (10 pg) plasmids were injected together with transposase mRNA into one-cell stage WT or snph db embryos. At 30-32 hpf, embryos were sorted for fluorescence, anesthetized in $0.015 \%$ tricaine, and embedded in a lateral view in 1\% low-melt agarose in E3 medium + PTU. Their right eye was removed using a pulled glass pipette with a short taper as described in previous studies (Poulain et al., 2010; Poulain and Chien, 2013; Gaynes et al., 2015). Of note, removal of the contralateral eye preserves the underlying neuroepithelium, which ensures that the optic tract environment is not changed by the surgery and that retinal axon elongation and guidance are not affected. Embryos were allowed to recover until $46 \mathrm{hpf}$, when they were reanesthetized and about one-third of the yolk was removed by squeezing it out through a small hole torn with sharpened tungsten needles. Embryos were allowed to recover, reanesthetized at 50-52 hpf, and remounted in a lateral view in 1\% low-melt agarose in E3 medium + PTU + tricaine in a membrane-bottomed Petri dish for time-lapse imaging on a Leica TCS SP8X laser-scanning confocal microscope equipped with LAS X software, HyD detectors, and a 40X objective (digital zoom 3, pinhole 1.25). Z-series were acquired for up to $3 \mathrm{~h}$ with $512 \times 512$ pixel resolution at 1 min intervals to minimize photobleaching. Z-intervals were $1 \mu \mathrm{m}$ with a $z$-range of $\sim 35-40 \mu \mathrm{m}$ to account for potential movement of the embryo. Chamber temperature was maintained at $28.5^{\circ} \mathrm{C}$. Maximal intensity projections for each time point were compiled and aligned using ImageJ software (Schindelin et al., 2012; Schneider et al., 2012; RRID: SCR_002285) and the StackReg plugin (Thévenaz et al., 1998). Kymograph analyses of mitochondrial movement were performed using the ImageJ plugins KymoToolBox (Zala et al., 2013; RRID:SCR_016098) and KymoAnalyzer (Neumann et al., 2017). The number of mitochondria arriving at and leaving the growth cone were counted manually from the time-lapse sequences.

For the visualization of Snphb at the growth cone, isl2b:mitoEGFP-2A-TagRFPCAAX (15 pg), isl2b:LifeActTagRFP (10 pg), and isl2b:TagBFP-snphb (25 pg) plasmids were coinjected with transposase mRNA at the one-cell stage. Embryos were prepared in the same manner as for time-lapse imaging (described above). Single $z$-stacks (z-interval of $0.42 \mu \mathrm{m}$ ) were acquired at $\sim 50-52$ hpf using a $40 \times$ objective with digital zoom 4 , pinhole 1.0 , and $1024 \times 1024$ pixel resolution.

For the visualization of mitochondria in single mature retinal axons at $120 \mathrm{hpf}$, embryos were injected with isl2b:mitoEGFP-2A-TagRFPCAAX (15 pg) and transposase mRNA at the one-cell stage and were selected for fluorescence in the optic tract at $96 \mathrm{hpf}$, after which the right eye was removed as described above. Larvae were reanesthetized at $120 \mathrm{hpf}$ and mounted in a lateral view in $1.5 \%$ low-melt agarose in E3 medium + PTU + tricaine in a membrane-bottomed Petri dish. Imaging was per- formed using a $40 \times$ objective with digital zoom 2.5 and pinhole 1.0. Time-lapse recordings were performed in 35 $z$-planes $(0.80 \mu \mathrm{m} \mathrm{z}$-interval), allowing acquisition at 1 frame every $15 \mathrm{~s}$ over $10 \mathrm{~min}$.

\section{Time-lapse image analysis}

Image analysis was conducted using ImageJ (Schindelin et al., 2012; Schneider et al., 2012). For 2D analyses, the TagRFP/TagRFPCAAX signals from stack maximal projections were used to manually segment the growth cone total and central areas. The growth cone central area was determined by tracing the perimeter of the growth cone body, not including filopodia. As such, the central area is an arbitrary outline based on growth cone morphology and fluorescent intensity that presumably corresponds to growth cone central domain and transition zone (it does not necessarily correspond to what is commonly defined as the growth cone central domain based on cytoskeletal components). Regions of interest (ROls) defined as the total and central areas were used to segment the mitoEGFP signals on binary images using the "particle analysis" tool. The following two different thresholds were used to analyze mitochondrial particles: a threshold of 55 was applied to the growth cone central area to segment the main mitochondrial cluster; and a threshold of 20 was applied to the growth cone total area to segment smaller individual mitochondria present in the peripheral area (Fig. 1B). A threshold of 20 was also applied to analyze pioneering mitochondrial clusters using the particle analysis tool. The number, area, and presence (percentage of time of advance) of pioneering mitochondrial clusters were analyzed in every frame of an advancing growth cone central area. The peripheral area, comprising filopodia, was calculated by subtracting the growth cone central area from the growth cone total area. The number of filopodia was counted manually. We drew a straight line from the mitochondrial cluster boundary to the most distal outline of the growth cone central area where two filopodia join together to measure the distance between the main mitochondrial cluster and the growth cone leading edge. We also defined the proximal growth cone as the most proximal boundary of the growth cone central area, where the growth cone transitions into the axon shaft. A growth cone was classified as advancing when the proximal growth cone advanced $>1 \mu \mathrm{m}$ after an elongation of the growth cone central area (i.e. major $>3 \times$ minor axis length of the best fitting ellipse of the central area). Elongation rates were quantified by measuring the displacement between the proximal growth cone boundaries before and after advance and expressed in micrometers per minute.

Visualizations of 3D growth cones and mitochondria were prepared using FluoRender (RRID:SCR_014303; Wan et al., 2017). For volumetric analysis of mitochondrial occupancy at the growth cone, $z$-stacks were processed and analyzed with ImageJ. Images were thresholded to the lowest level that excludes the majority of noise pixels to obtain binary $z$-projections similar to raw $z$-projections, after which volumes were calculated using the Voxel Counter plugin. Volume data of mitochondria and the 
A
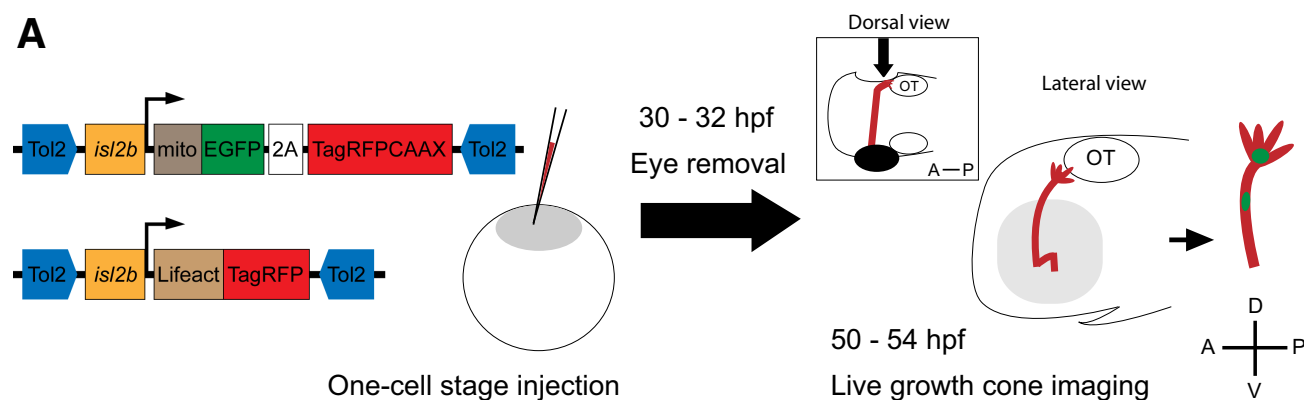

B

One-cell stage injection

Live growth cone imaging

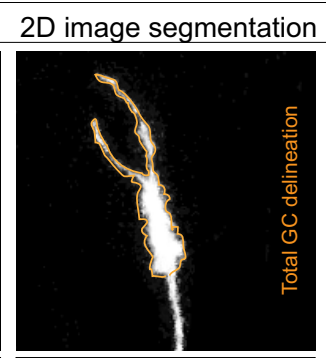

\section{GC classification}

Pausing GC

Advancing GC
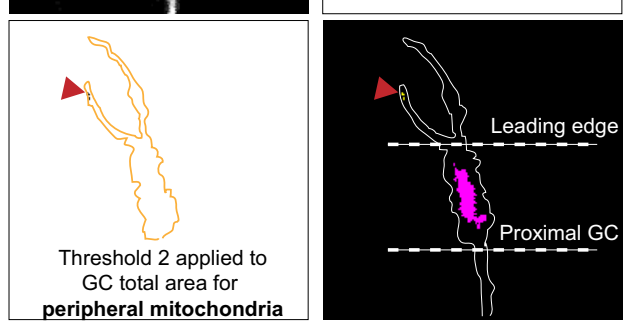

C

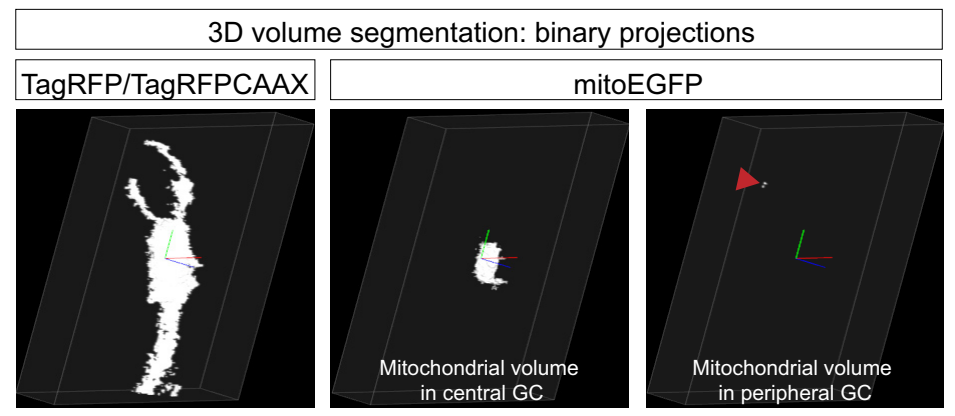

Figure 1. Live imaging approach and analysis. A, Individual retinal axons and mitochondria were mosaically labeled by coinjecting isl2b:mitoEGFP-2A-TagRFPCAAX and isl2b:Lifeact-TagRFP plasmids at the one-cell stage. isl2b:Lifeact-TagRFP was used to improve the visualization of single axons and growth cone (GC) filopodia (for more details, see main text and Materials and Methods). After removal of the contralateral eye, axons growing along the optic tract toward the optic tectum (OT) and their mitochondria were imaged in a lateral view between 50 and $54 \mathrm{hpf}(\Delta t=1 \mathrm{~min})$. A, Anterior; D, dorsal; OT, optic tectum; P, posterior; V, ventral. B, Growth cone total and central areas visualized with TagRFP/TagRFPCAAX were manually segmented and used as ROIs for segmenting mitochondrial signals using the particle analysis tool in ImageJ. Two different thresholds were used to analyze mitochondrial particles: a threshold of 55 was applied to the growth cone central area to segment the main mitochondrial cluster, and a threshold of 20 was applied to the growth cone total area to segment smaller individual mitochondria present in the peripheral area (red arrowheads). Time-lapse recordings were classified according to growth cone behavior. Growth cone leading edge and proximal growth cone are indicated in the merged image; see Materials and Methods for definitions. Lateral view, confocal maximal projections. Scale bar, $5 \mu \mathrm{m}$. C, Volumes of mitochondria and GC total and central volumes were calculated using 3D analysis. GC total and central volumes visualized with TagRFP/TagRFPCAAX were calculated on binary z-projections and used as volumes of interest for segmenting mitochondrial volumes using the voxel counter plugin in ImageJ (see also Movie 1).

growth cone, expressed in cubic micrometers, were divided to obtain percentages of occupancy. Figures were prepared using Adobe Photoshop and Illustrator, and time-lapse videos were assembled using ImageJ.

\section{Statistics}

Data were analyzed and graphs were produced using Prism (GraphPad Software). Data are presented as the mean \pm SEM. Statistical tests were applied as indicated 
Table 2: Summary of statistical analyses

\begin{tabular}{|c|c|c|c|c|c|}
\hline Figure & Measurement & Data structure & Type of test & Comparison & Statistical value \\
\hline Fig. $2 B$ & Mitochondrial occupancy (GC total volume) & Normal & Unpaired $t$ test & Pausing vs advancing GC & $\begin{array}{l}p=0.4065 \\
t_{(17)}=0.8511\end{array}$ \\
\hline Fig. 2B' & Mitochondrial occupancy (GC total area) & Normal & Unpaired $t$ test & Pausing vs advancing GC & $\begin{array}{l}p=0.4310 \\
t_{(17)}=0.8067\end{array}$ \\
\hline Fig. $2 C$ & Mitochondrial occupancy (GC central volume) & Normal & Unpaired $t$ test & Pausing vs advancing GC & $\begin{array}{l}p=0.2829 \\
t_{(17)}=1.109\end{array}$ \\
\hline Fig. $2 C^{\prime}$ & Mitochondrial occupancy (GC central area) & Normal & Unpaired $t$ test & Pausing vs advancing GC & $\begin{array}{l}p=0.2325 \\
t_{(17)}=1.238\end{array}$ \\
\hline Fig. $2 D$ & Mitochondrial volume (peripheral volume) & Normal & Unpaired $t$ test & Pausing vs advancing GC & $\begin{array}{l}p=0.4444 \\
t_{(17)}=0.7831\end{array}$ \\
\hline Fig. $2 D^{\prime}$ & Number of mitochondria in GC peripheral area & Normal & Unpaired $t$ test & Pausing vs advancing GC & $\begin{array}{l}p=0.7028 \\
t_{(15)}=0.3889\end{array}$ \\
\hline Fig. $2 E$ & Distance from leading edge & Normal & Unpaired $t$ test & Pausing vs advancing GC & $\begin{array}{l}p<0.0001 \\
t_{(17)}=6.740\end{array}$ \\
\hline Fig. $4 B$ & Percentage net mitochondrial transport & Normal & $\begin{array}{l}\text { Two-way ANOVA } \\
\text { (post hoc Bonferroni) }\end{array}$ & Pausing vs advancing GC & $\begin{array}{l}p=0.9955 \\
F_{(1,34)}=0.00003\end{array}$ \\
\hline Fig. $4 C$ & Percentage time mitochondria spent mobile & Normal & Unpaired $t$ test & Pausing vs advancing GC & $\begin{array}{l}p=0.0131 \\
t_{(17)}=2.772\end{array}$ \\
\hline Fig. $4 C$ & Percentage time mitochondria spent stationary & Normal & Unpaired $t$ test & Pausing vs advancing GC & $\begin{array}{l}p=0.0075 \\
t_{(17)}=3.034\end{array}$ \\
\hline Fig. $4 D$ & Mitochondrial flux (arriving mitochondria) & Normal & Unpaired $t$ test & Pausing vs advancing GC & $\begin{array}{l}p=0.4666 \\
t_{(17)}=0.7448\end{array}$ \\
\hline Fig. $4 D$ & Mitochondrial flux (leaving mitochondria) & Normal & Unpaired $t$ test & Pausing vs advancing GC & $\begin{array}{l}p=0.3617 \\
t_{(17)}=0.9374\end{array}$ \\
\hline Fig. $4 E$ & Number of arriving mitochondria vs axon growth & Normal & Linear regression & & $\begin{array}{l}p=0.0071 \\
r^{2}=0.5324\end{array}$ \\
\hline Fig. $5 D$ & snpha expression & Normal & Unpaired $t$ test & WT vs lak & $\begin{array}{l}p<0.0001 \\
t_{(4)}=18.33\end{array}$ \\
\hline Fig. $5 D$ & snphb expression & Normal & Unpaired $t$ test & WT vs lak & $\begin{array}{l}p<0.0001 \\
t_{(4)}=17.84\end{array}$ \\
\hline Fig. $6 E$ & Percentage stationary mitochondria & Normal & Unpaired $t$ test & WT vs snph $d b$ & $\begin{array}{l}p=0.0074 \\
t_{(13)}=3.168\end{array}$ \\
\hline Fig. $7 A$ & $\begin{array}{l}\text { GC total occupancy } \\
\text { (percentage volume) }\end{array}$ & Normal & Unpaired $t$ test & WT vs snph $d b$ & $\begin{array}{l}p=0.0023 \\
t_{(22)}=3.446\end{array}$ \\
\hline Fig. $7 A^{\prime}$ & $\begin{array}{l}\text { GC total occupancy } \\
\text { percentage area) }\end{array}$ & Normal & Unpaired $t$ test & WT vs snph $d b$ & $\begin{array}{l}p=0.0178 \\
t_{(22)}=2.562\end{array}$ \\
\hline Fig. $7 B$ & $\begin{array}{l}\text { GC central occupancy } \\
\text { (percentage volume) }\end{array}$ & Normal & Unpaired $t$ test & WT vs snph $d b$ & $\begin{array}{l}p=0.0184 \\
t_{(22)}=2.543\end{array}$ \\
\hline Fig. $7 B^{\prime}$ & $\begin{array}{l}\text { GC central occupancy } \\
\text { (percentage area) }\end{array}$ & Normal & Unpaired $t$ test & WT vs snph $d b$ & $\begin{array}{l}p=0.0071 \\
t_{(22)}=2.967\end{array}$ \\
\hline Fig. $7 C$ & Peripheral mitochondrial volume & Normal & Unpaired $t$ test & WT vs snph $d b$ & $\begin{array}{l}p=0.2765 \\
t_{(22)}=1.116\end{array}$ \\
\hline Fig. $7 C^{\prime}$ & Number of mitochondria in peripheral area & Normal & Unpaired $t$ test & WT vs snph $d b$ & $\begin{array}{l}p=0.6030 \\
t_{(21)}=0.5278\end{array}$ \\
\hline Fig. $7 D$ & Distance from leading edge (pausing GC) & Normal & Unpaired $t$ test & WT vs snph $d b$ & $\begin{array}{l}p=0.0003 \\
t_{(20)}=4.403\end{array}$ \\
\hline Fig. $7 D^{\prime}$ & Distance from leading edge (advancing GC) & Normal & Unpaired $t$ test & WT vs snph $d b$ & $\begin{array}{l}p=0.1080 \\
t_{(16)}=1.703 \\
p=0.4976\end{array}$ \\
\hline Fig. $7 E$ & $\begin{array}{l}\text { Mitochondrial flux } \\
\text { (arriving mitochondria) }\end{array}$ & Normal & Unpaired $t$ test & WT vs snph $d b$ & $\begin{array}{l}p=0.4976 \\
t_{(22)}=0.6904\end{array}$ \\
\hline Fig. $7 E$ & $\begin{array}{l}\text { Mitochondrial flux } \\
\text { (leaving mitochondria) }\end{array}$ & Normal & Unpaired $t$ test & WT vs snph $d b$ & $\begin{array}{l}p=0.0412 \\
t_{(22)}=2.168\end{array}$ \\
\hline Fig. $7 F$ & Percentage net mitochondrial transport (anterograde) & Normal & Unpaired $t$ test & WT vs snph $d b$ & $\begin{array}{l}p=0.0178 \\
t_{(22)}=2.561\end{array}$ \\
\hline Fig. $7 F$ & Percentage NET mitochondrial transport (retrograde) & Normal & Unpaired $t$ test & WT vs snph $d b$ & $\begin{array}{l}p=0.0529 \\
t_{(22)}=2.046\end{array}$ \\
\hline Fig. $7 G$ & Percentage time mitochondria spent in motion (anterograde) & Normal & Unpaired $t$ test & WT vs snph $d b$ & $\begin{array}{l}p=0.3330 \\
t_{(22)}=0.9900\end{array}$ \\
\hline Fig. $7 G$ & Percentage time mitochondria spent in motion (retrograde) & Normal & Unpaired $t$ test & WT vs snph $d b$ & $\begin{array}{l}p=0.5596 \\
t_{(22)}=0.5924\end{array}$ \\
\hline Fig. $7 H$ & $\mathrm{GC}$ total area & Normal & Unpaired $t$ test & WT vs snph $d b$ & $\begin{array}{l}p=0.2904 \\
t_{(22)}=1.083\end{array}$ \\
\hline Fig. $7 H$ & GC central area & Normal & Unpaired $t$ test & WT vs snph $d b$ & $\begin{array}{l}p=0.3869 \\
t_{(22)}=0.8829\end{array}$ \\
\hline Fig. $7 I$ & Number of filopodia & Normal & Unpaired $t$ test & WT vs snph $d b$ & $\begin{array}{l}p=0.6775 \\
t_{(22)}=0.42154\end{array}$ \\
\hline Fig. $7 J$ & Growth rate & Normal & Unpaired $t$ test & WT vs snph $d b$ & $\begin{array}{l}p=0.8256 \\
t_{(15)}=0.2243\end{array}$ \\
\hline Fig. $8 D$ & Pioneering cluster area & Normal & Unpaired $t$ test & WT vs snph $d b$ & $\begin{array}{l}p=0.0166 \\
t_{(15)}=2.697\end{array}$ \\
\hline Fig. $8 E$ & Number of pioneering clusters & Normal & Unpaired $t$ test & WT vs snph $d b$ & $\begin{array}{l}p=0.1865 \\
t_{(15)}=1.385\end{array}$ \\
\hline Fig. $8 F$ & Mitochondria presence (\% time) & Normal & Unpaired $t$ test & WT vs snph $d b$ & $\begin{array}{l}p=0.1994 \\
t_{(15)}=1.343\end{array}$ \\
\hline
\end{tabular}




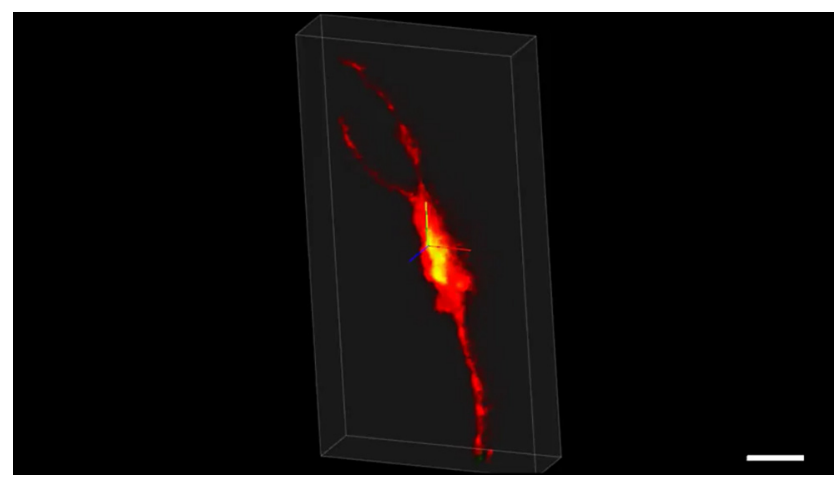

Movie 1. Representative $3 D$ visualization of mitochondria in a pausing growth cone. Video corresponding to Figure $1 \mathrm{C}$ showing mitochondria (green) in a distal retinal axon and growth cone (red) pausing along the optic tract. Scale bar, $5 \mu \mathrm{m}$. [View online]

in the Results and figure legends. Additional statistical details are provided in Table 2. Normal distribution was determined by column test.

\section{Results}

\section{Mitochondrial localization at the growth cone correlates with axon outgrowth}

To monitor mitochondrial behavior in developing axons in vivo, we performed high-resolution confocal time-lapse imaging of mitochondria in retinal axons elongating along the optic tract of zebrafish embryos between 50 and 55 hpf (Fig. 1A). We coexpressed EGFP targeted to mitochondria (mitoEGFP) and TagRFP targeted to the plasma membrane (TagRFPCAAX), and to actin filaments (LifeactTagRFP) in single retinal axons, and monitored labeled axons elongating along the contralateral optic tract toward the optic tectum (Fig. 1, Movie 1). As described previously (Bovolenta and Mason, 1987; Holt and Harris, 1993), growth cones of retinal axons in the tract were slender with numerous filopodia protruding and retracting dynamically. We could distinguish two classes of axons depending on their outgrowth status (Fig. 2A). Advancing axons had very elongated growth cones, whereas pausing, not growing, axons had rounder growth cones with filopodia oriented in all directions, supporting the notion that growth cone shape correlates with growth cone behavior (Bovolenta and Mason, 1987; Mason and Wang, 1997). For the duration of our time-lapse videos (range, 30-160 min), several growth cones were found to alternate between pausing and advancing behaviors and could therefore be classified into both categories (Movies $2,3,4)$.

As previously observed in vitro (Morris and Hollenbeck, 1993), mitochondria were abundantly detected at the growth cone (Fig. 2A, Movies 1, 2, 3, 4). The majority of mitochondria clustered in the microtubule-rich growth cone central area, whereas smaller mitochondria regularly appeared in the peripheral area along protrusions or actin filopodia (Movie 3). We analyzed mitochondrial localization and distribution at the growth cone by quantifying mitochondrial occupancy in the growth cone total and central volumes (Fig. 2B,C), peripheral mitochondrial vol- ume (Fig. 2D), mitochondrial occupancy in the growth cone total and central areas (Fig. $2 B^{\prime}, C^{\prime}$ ), the number of mitochondria in the peripheral area (Fig. 2D), and the distance between mitochondria and the growth cone leading edge (Fig. 2E; see also Fig. 1B,C). Mitochondrial occupancy was chosen as a measure to evaluate mitochondrial density in the growth cone since spatial overlap of mitochondria within the growth cone central zone did not allow the visualization and quantification of single mitochondria. Mitochondrial occupancy in the total and central volumes and areas were similar in advancing versus pausing growth cones (Fig. $2 B-C$ ). We did note that when we exclusively compared growth cones that frequently alternated between pausing and advancing behaviors, a significant decrease in mitochondrial occupancy in the central area of advancing growth cones was observed (paired $t$ test, $p=0.006, t_{(6)}=4.2$ ). Furthermore, the distance of the main mitochondrial cluster to the leading edge was significantly different between pausing and advancing growth cones (Fig. 2E). When a growth cone advanced, its central area elongated substantially, but this forward extension was not accompanied with a forward advance of mitochondria (Fig. $3 A$, Movie 5). Hence, the distance between them and the leading edge increased twofold (Fig. 2E). Mitochondria repositioned near the leading edge when the growth cone regained a rounder shape (Fig. $3 A$ ), which often indicated a transition to a pausing state. Interestingly, although the majority of mitochondria lagged behind during growth cone extension, a smaller subset of mitochondria was always detected adjacent to the leading edge (Fig. 3, Movie 5). We analyzed the number and area of these "pioneering mitochondrial clusters," as well as the amount of time they were present when a growth cone advanced. We found that pioneering mitochondrial clusters had on average a total area of $1.1 \pm 0.4 \mu \mathrm{m}^{2}$, and that $2.6 \pm 0.3$ mitochondrial clusters positioned near the leading edge in $43 \pm$ $9.5 \%$ of the time of advance. Importantly, pioneering mitochondria were consistently observed in every growth cone that elongated.

Altogether, our data show that mitochondria localize in the growth cone central area in vivo and are also present along filopodia extending from the growth cone periphery. The position of mitochondria in the growth cone central area further changes with the growth status of the axon.

\section{Mitochondrial transport is coordinated with growth cone behavior and axon growth in vivo}

Next, we asked whether transport of mitochondria in the axon shaft proximal to the growth cone also relates to growth status. We performed kymograph analysis to measure net mitochondrial transport as well as the percentage of time mitochondria spent in motion (Fig. 4A-C). In agreement with a higher mobility of mitochondria observed in developing versus mature axons (Lewis et al., 2016), $<5 \%$ of all mitochondria were stationary (Fig. 4C). The majority of mobile mitochondria moved anterogradely, with a significantly higher percentage of anterograde versus retrograde trafficking in both pausing and advancing growth cones (Fig. 4B). An analysis of mito- 
A
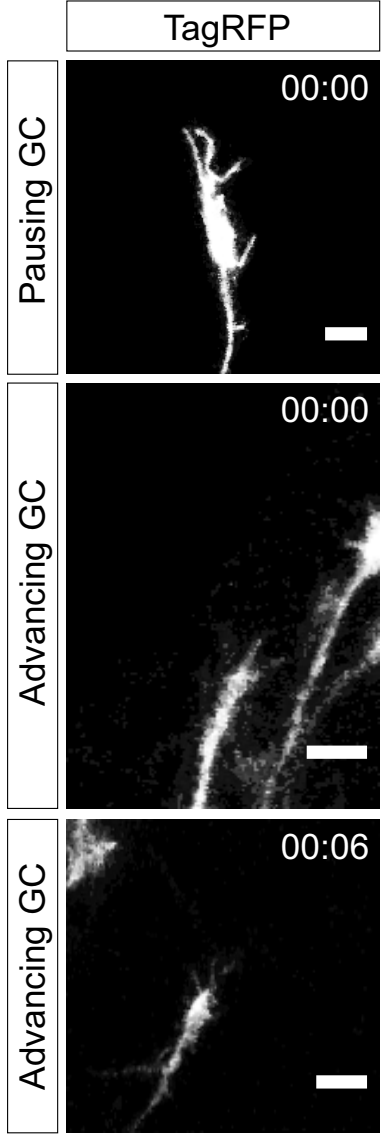

B

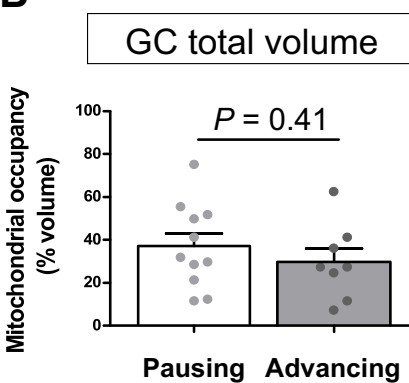

B'

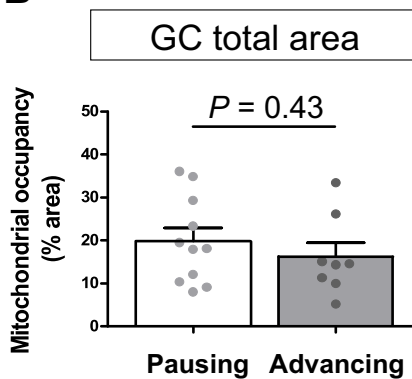

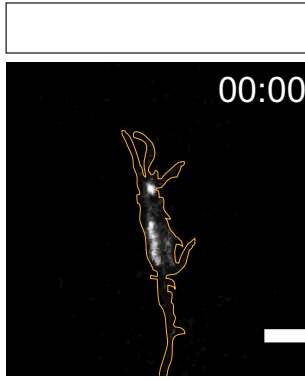
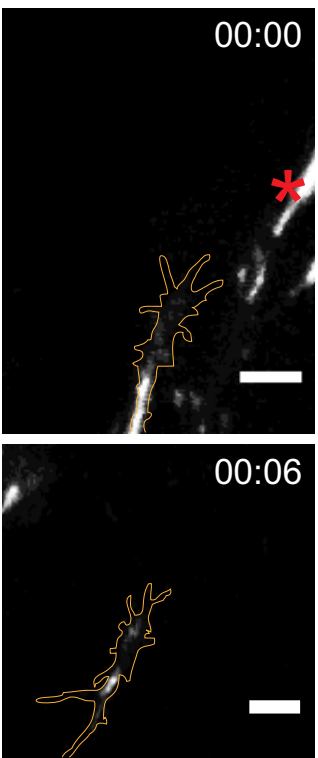

C

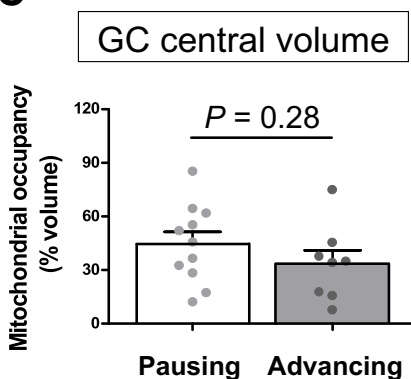

C'

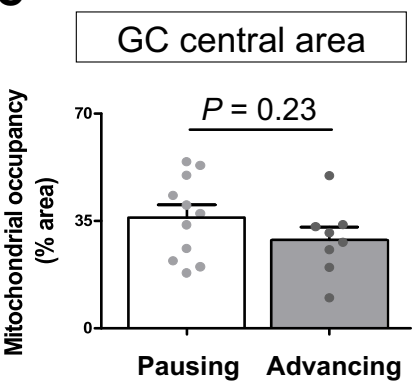

mitoEGFP
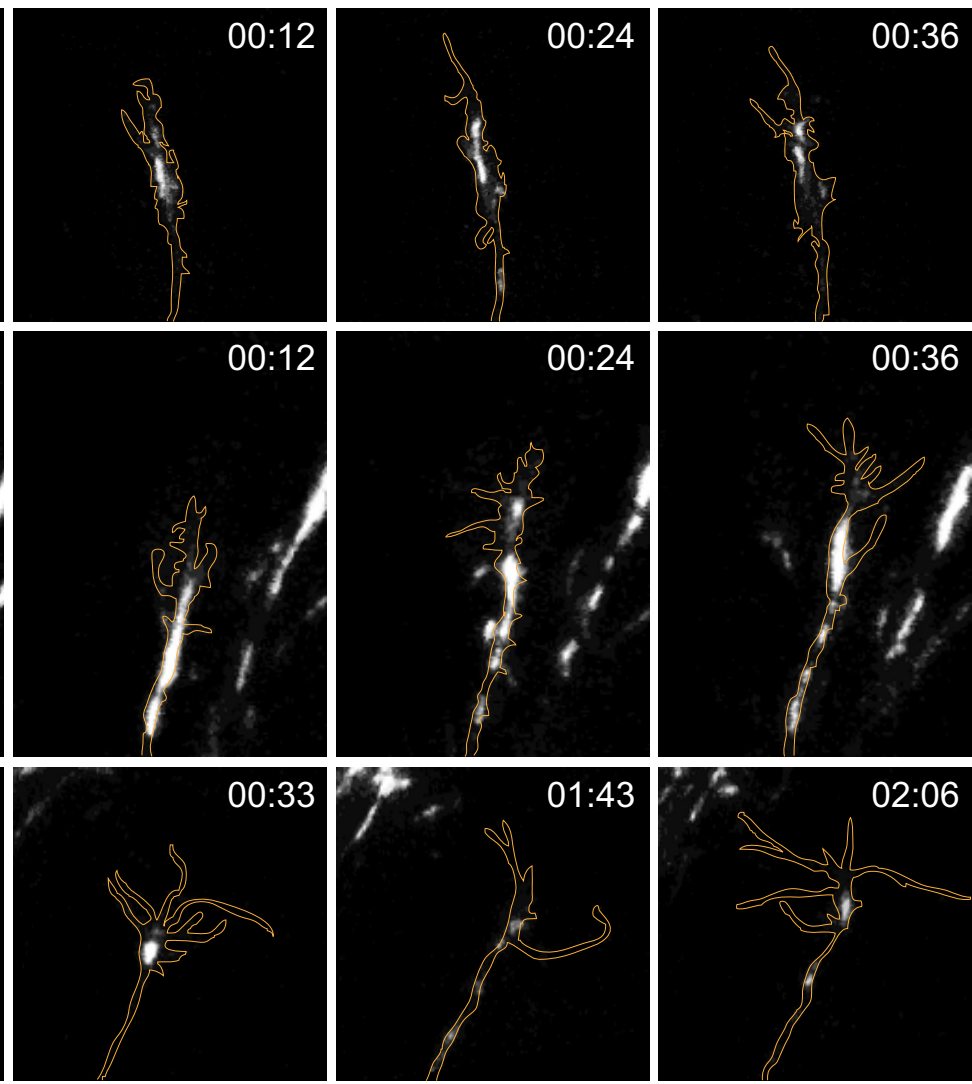

D

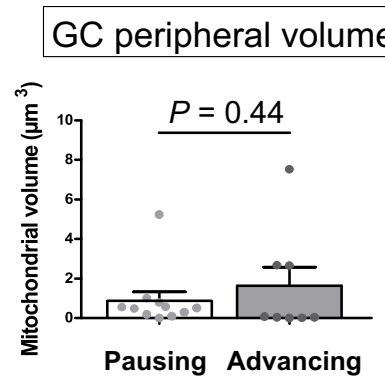

E

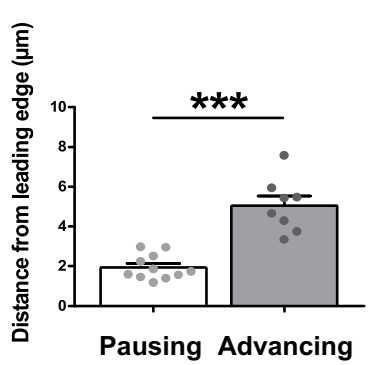

D'

GC peripheral area

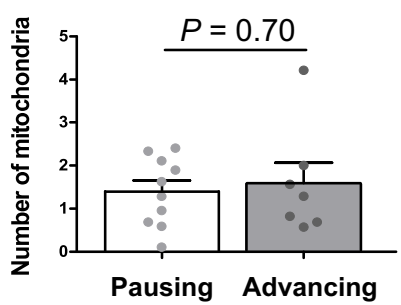

Figure 2. Mitochondrial distribution changes with growth cone behavior. $\boldsymbol{A}$, Representative time-lapse images of mitochondria (mitoEGFP, white) in distal retinal axons and growth cones (labeled with TagRFP, delineated in orange and shown in left panels) classified as pausing or advancing (see Movies 2, 3, 4). Most growth cones alternate periods of pausing and advancing/elongating, resulting in net axon growth, as shown in the bottom panels. The red asterisk indicates mitochondria in neighboring axons. Scale bar, $5 \mu \mathrm{m}$. B-C ', Quantification of the mitochondrial occupancy (as a percentage) of the growth cone total volume (B), total area $(\boldsymbol{B})$, central volume $(\boldsymbol{C})$, and central area $(\boldsymbol{C})$. $\boldsymbol{D}, \boldsymbol{D}^{\prime}$, Quantification of the mitochondrial volume in the peripheral growth cone $(\boldsymbol{D})$ and of the number of mitochondria in the growth cone peripheral area $(\boldsymbol{D})$. $\boldsymbol{E}$, Quantification of the distance between the largest 
continued

mitochondrial cluster and the growth cone leading edge. When growth cones elongate, the distance to the growth cone leading edge increases significantly. Data from 12 independent experiments (pausing, $n=11$; advancing, $n=8$ ) are shown as mean \pm SEM. Statistical analysis $(\boldsymbol{B}-\boldsymbol{E})$ : unpaired $t$ test, $* * * p<0.001$

chondrial transport over shorter timescales showed a similar proportion of mitochondria moving anterogradely (71 $\pm 5.4 \%$ in long movies, $78 \pm 7.3 \%$ over shorter timescales), retrogradely ( $26 \pm 4.5 \%$ in long movies, $20 \pm$ $6.7 \%$ over shorter timescales), or remaining stationary $(2.3 \pm 1.6 \%$ in long movies, $2.3 \pm 2.3 \%$ over shorter

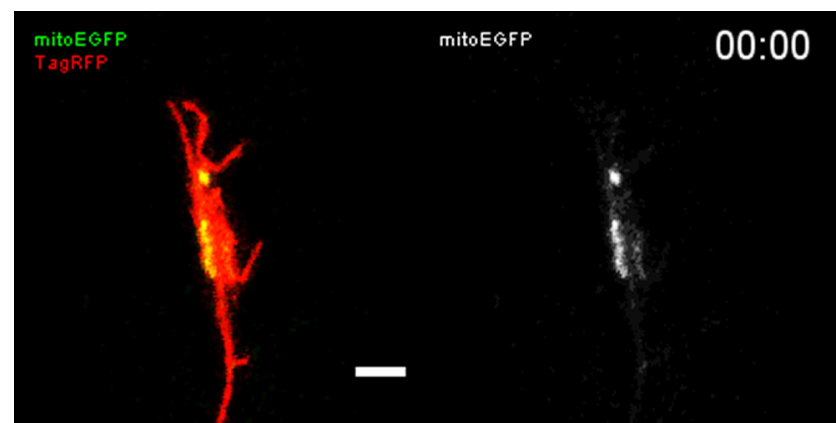

Movie 2. Representative time-lapse recording of mitochondrial dynamics in an advancing growth cone. Time-lapse video corresponding to Figure $2 A$ showing mitochondria (green, white) in a distal retinal axon and growth cone (red) elongating along the optic tract. Images were acquired at 1 min intervals for 48 min. Each frame is a confocal image stack maximal projection, lateral view, and anterior is on the left. Time stamp format: hours: minutes (hr:min). Scale bar, $5 \mu \mathrm{m}$. [View online]

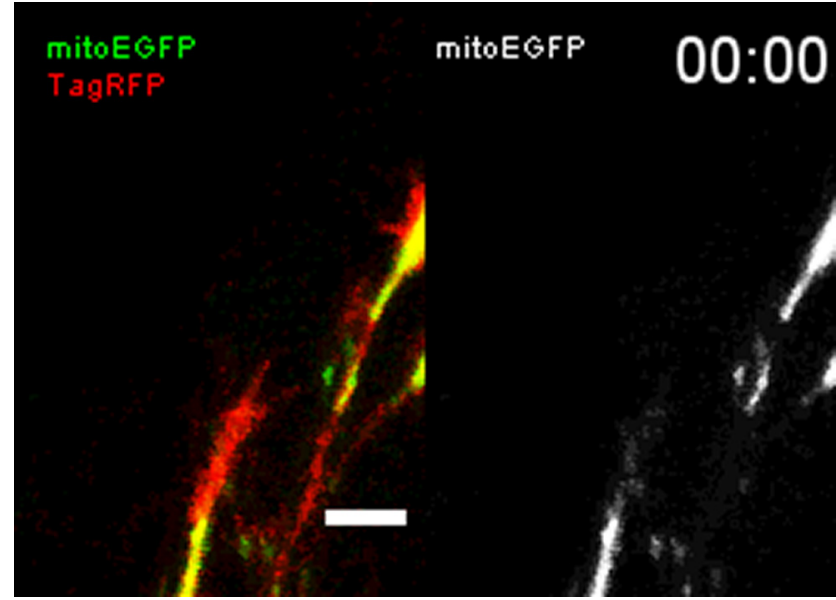

Movie 3. A subset of mitochondria localizes to the leading edge of the growth cone during elongation. Representative time-lapse video corresponding to Figure $3 A$ showing mitochondria (green, white) in a distal retinal axon and growth cone (red) elongating along the optic tract. Images were acquired at $1 \mathrm{~min}$ intervals for $36 \mathrm{~min}$. Note that the growth cone is elongating from $00: 10$ to $00: 25$. During this elongation, most mitochondria lag behind, but a subset of smaller mitochondria localizes adjacent to the leading edge. Each frame is a confocal image stack maximal projection, lateral view, and anterior is on the left. Time stamp format: hours:minutes (hr:min). Scale bar, $3 \mu \mathrm{m}$. [View online] timescales), indicating that the length of time-lapse movies had no effect on mitochondrial transport parameters (two-way ANOVA, effect of time, $p=0.9994, F_{(1.66)}<$ 0.0001). Mitochondria spent more time moving when a growth cone advanced (Fig. 4C), and we observed a trend toward more mitochondria arriving versus leaving in both pausing and advancing growth cones (Fig. 4D). We finally found a strong correlation between the number of mitochondria arriving at the growth cone and the distance the axon elongated (Fig. 4E). Altogether, these results illustrate that mitochondrial transport toward the growth cone correlates with growth cone advance, suggesting that mitochondrial motility and axon elongation may be functionally linked or coregulated in vivo.

\section{Zebrafish Syntaphilins are expressed in RGCs and localize to the growth cone}

How mitochondria are maintained in the growth cone remains poorly understood. One possible mechanism involves the attachment, or docking, of mitochondria to microtubules by Snph. Snph was identified as an outer mitochondrial membrane protein that is able to dock mitochondria directly to microtubules via its microtubulebinding domain (MTB; Kang et al., 2008; see Fig. 6A). Snph was shown to be important in axons for the regulation of branching (Courchet et al., 2013) and synaptic plasticity (Kang et al., 2008). We decided to test whether Snph also plays a role in docking mitochondria at the growth cone during axon elongation in vivo. Due to the whole-genome duplication that occurred in the teleost lineage (Glasauer and Neuhauss, 2014), two snph genes, snpha and snphb, are present in zebrafish. Both Snpha and Snphb share a high sequence conservation with human SNPH in their MTB and mitochondrial transmembrane domains (TMs), strongly suggesting a conserved mitochondrial anchoring function (see Fig. $6 A$, Extended Data Fig. 6-1). We first analyzed the expression of snpha

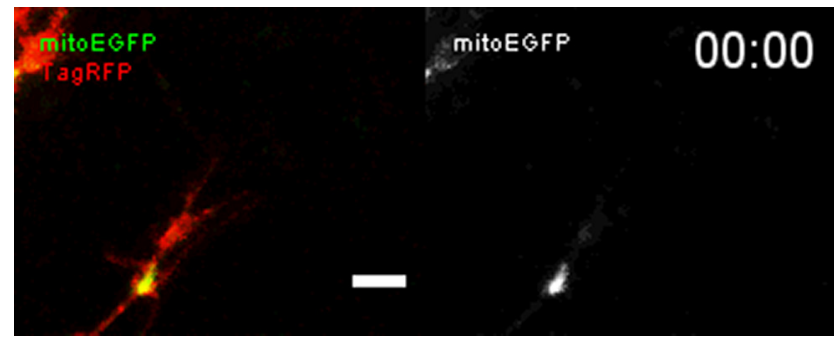

Movie 4. Representative time-lapse recording of mitochondrial dynamics in a pausing growth cone. Time-lapse video corresponding to Figure $2 A$ showing mitochondria (green, white) in a distal retinal axon and growth cone (red) pausing along the optic tract. Images were acquired at $1 \mathrm{~min}$ intervals for $48 \mathrm{~min}$. Each frame is a confocal image stack maximal projection, lateral view, and anterior is on the left. Time stamp format: hours:minutes (hr:min). Scale bar, $5 \mu \mathrm{m}$. [View online] 
A
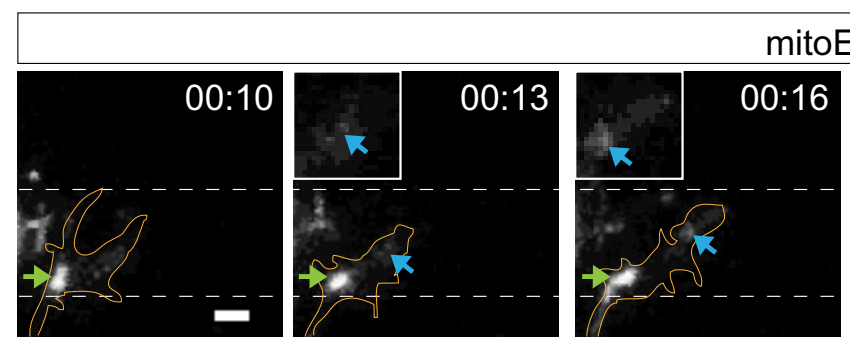

mitoEGFP
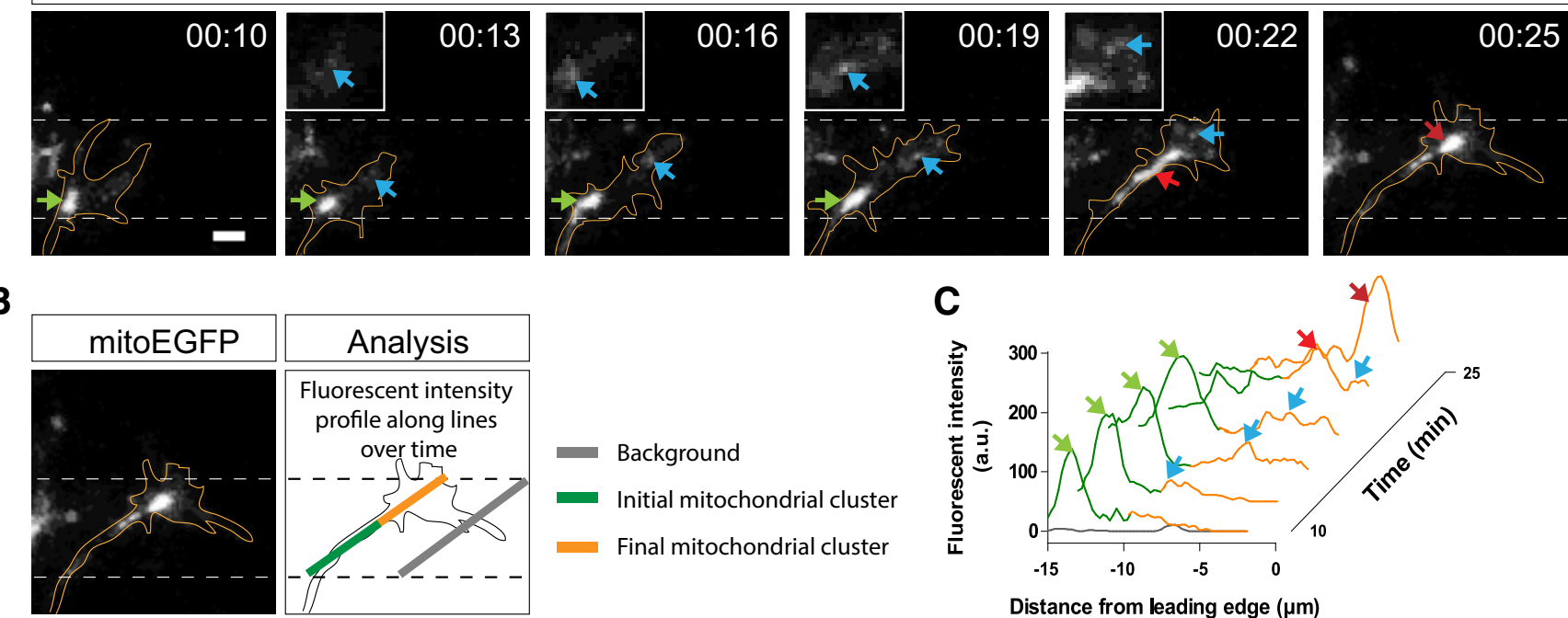

Figure 3. Pioneering mitochondrial clusters localize near the leading edge in advancing growth cones. A, Representative time-lapse images of mitochondria (mitoEGFP, white) in an advancing growth cone (Movie 5). While the main mitochondrial cluster (green arrow) lags behind during growth cone advance, some small pioneering mitochondrial clusters (blue arrows) appear in close proximity to the leading edge. Lateral view, confocal maximal projections. Scale bar, $3 \mu \mathrm{m}$. $\boldsymbol{B}, \boldsymbol{C}$, EGFP intensity profiles calculated along a line between the initial proximal growth cone and final leading edge to analyze the distribution of mitochondria along the advancing growth cone at successive time points shown in $\boldsymbol{A}$. $\boldsymbol{C}$, The orange and green lines correspond to the fluorescent intensities of mitochondria in the growth cone and at the proximal growth cone, respectively (see $\boldsymbol{B}$ : Analysis).

and snphb during zebrafish development. Quantification of snpha and snphb mRNA levels using reverse transcriptase (RT)-coupled ddPCR showed that both snpha and snphb are maternally expressed and that mRNA levels for both snphs increase over later developmental periods, albeit at much lower levels for snpha (Fig. 5A). ISH further revealed a high expression of snphb in the nervous system at 48, 72, and $120 \mathrm{hpf}$ (Fig. 5B), whereas snpha expression was not detectable using this approach (data not shown). Importantly, snphb was detected in the RGC

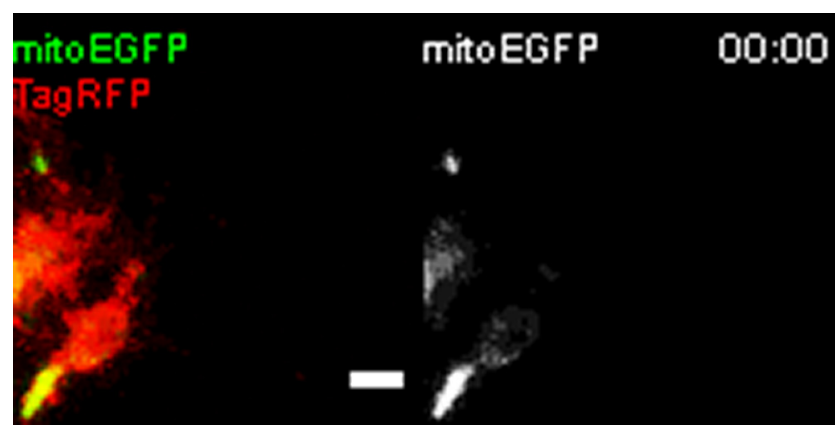

Movie 5. Representative time-lapse recording of mitochondrial dynamics in a growth cone that combines pausing and advancing. Time-lapse video corresponding to Figure $2 A$ showing mitochondria (green, white) in a distal retinal axon and growth cone (red) that alternates between pausing and elongating behaviors. The growth cone is pausing during the majority of the recording, but advances intermittently (e.g., from 00:00 to 00:18, 01:39 to 01:55, and 02:24 to 02:39). Images were acquired at $1 \mathrm{~min}$ intervals for $160 \mathrm{~min}$. Each frame is a confocal image stack maximal projection, lateral view, and anterior is on the left. Time stamp format: hours:minutes (hr:min). Scale bar, $5 \mu \mathrm{m}$. [View online] layer at the time of retinal axon elongation (Fig. 5B), suggesting a possible role in anchoring mitochondria in retinal growth cones. To further test whether snphb is expressed by RGCs, we performed ISH on WT and RGCdeficient lak mutant embryos (Kay et al., 2001) at $72 \mathrm{hpf}$ (Fig. 5C). Snphb expression was strongly reduced in the retina of lak compared with WT, while it appeared unchanged in the brain (Fig. $5 \mathrm{C}$ ). This decreased retinal expression was further confirmed by RT-ddPCR performed on dissected eyes (Fig. 5D). Snphb mRNA levels were highly detected in WT but significantly reduced in lak, indicating that snphb is strongly expressed by RGCs. Interestingly, snpha transcripts were also detected in WT, albeit at much lower levels. Like snphb, snpha eye expression was significantly decreased in lak (Fig. 5D), indicating that both Snphs are present in RGCs at the time of axon elongation.

As snphb is highly expressed in RGCs, we finally tested whether Snphb could localize to the growth cones of retinal axons elongating along the tract. As we could not identify an antibody directed against mammalian Snph that would specifically recognize zebrafish Snphs (data not shown), we decided to coexpress TagBFP-tagged Snphb, mitoEGFP, TagRFPCAAX, and Lifeact-TagRFP in single retinal axons, and to monitor labeled growth cones advancing along the contralateral optic tract (Fig. 5E). We detected TagBFP-Snphb in the central area of retinal growth cones, where it appeared to colocalize with the main mitochondrial cluster. Altogether, our results show that Snphs are expressed by RGCs and can localize to the growth cone of developing axons, suggesting they might participate to mitochondria docking at the growth cone during axon elongation in vivo. 
A
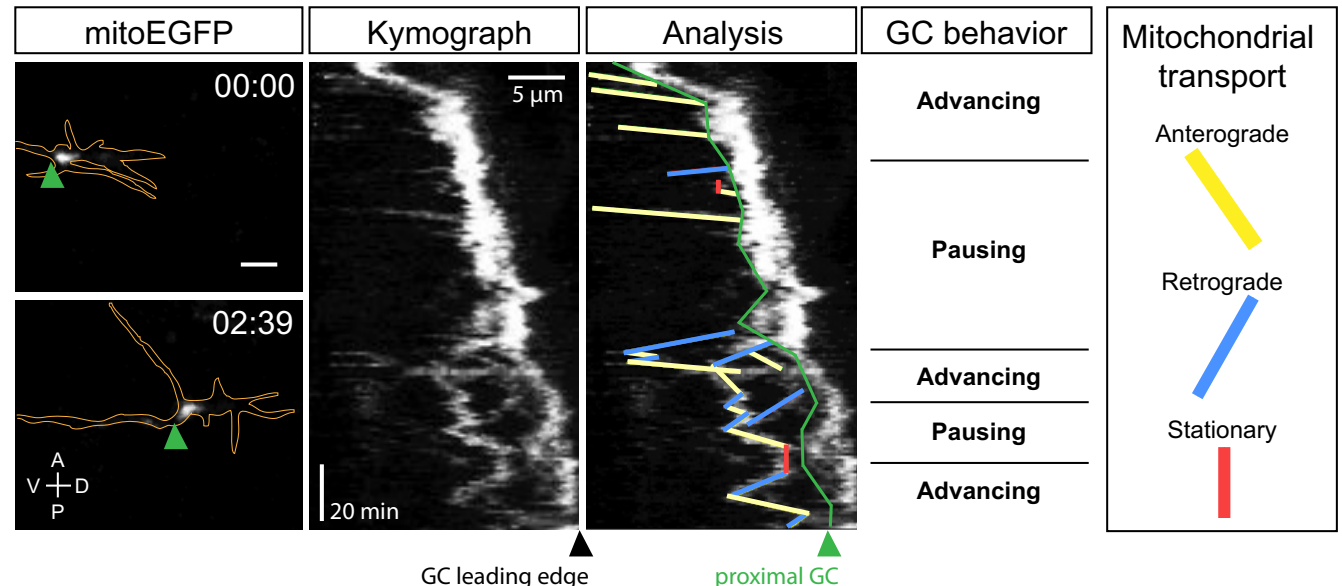

GC leading edge

proximal GC
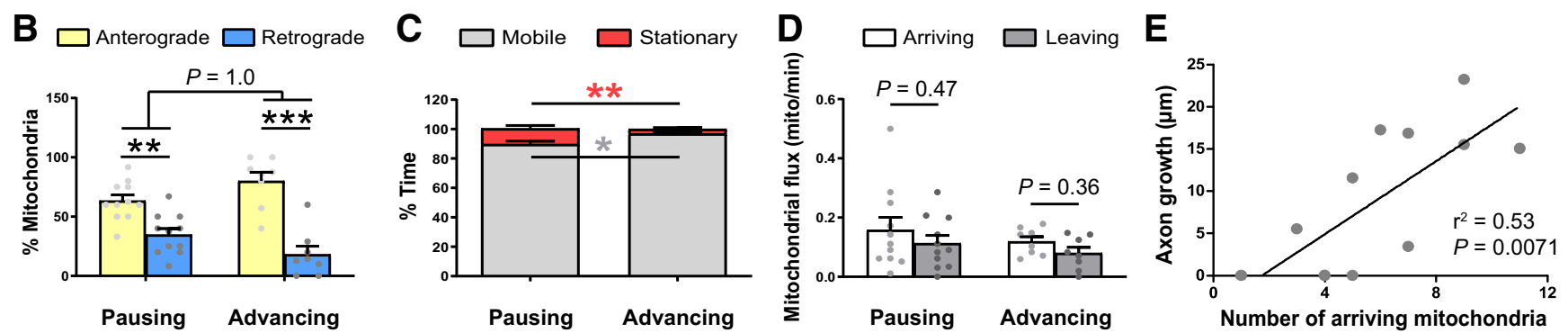

Figure 4. Anterograde mitochondrial transport correlates with axonal outgrowth. $\boldsymbol{A}$, Representative kymograph of mitochondria (mitoEGFP, white) in a distal axon whose growth cone alternates between advancing and pausing. The first and last frames of the time-lapse recording (Movie 4) are shown with axon and growth cone delineated in orange. Confocal maximal projections. Scale bar, $5 \mu \mathrm{m}$. The growth cone leading edge and proximal growth cone (green line) are indicated on the kymograph and kymograph analysis panels. $\boldsymbol{B}$, Quantification of net transport, analyzed by counting the number of mitochondria moving anterogradely or retrogradely. Data from 12 independent experiments (pausing: $n=11$, advancing: $n=8$ ) are shown as the mean \pm SEM. Statistical analysis: two-way ANOVA with post hoc Bonferroni test, $* * p<0.01$, $* * * p<0.001$. $\boldsymbol{C}$, Quantification of the percentage of time mitochondria spent in a mobile or stationary state. A significant increase in time spent in motion (grey asterisk, $p=0.013$ ) and a decrease in time spent in a stationary state (red asterisk, $p=0.008$ ) are observed proximally to growth cones that are advancing. Data from 12 independent experiments (pausing, $n=11$; advancing, $n=8$ ) are shown as the mean \pm SEM. Statistical analysis: unpaired $t$ test. $\boldsymbol{D}$, Quantification of mitochondrial flux, showing a trend toward more mitochondria arriving versus leaving the growth cone in both pausing and advancing growth cones. Data from 12 independent experiments (pausing, $n=11$; advancing, $n=8$ ) are shown as the mean \pm SEM. Statistical analysis: unpaired $t$ test. $\boldsymbol{E}$, Linear regression analysis between the number of mitochondria arriving at a growth cone and growth cone advance $(n=12)$.

\section{Syntaphilins contribute to mitochondrial immobilization at the growth cone}

To test the function of zebrafish Snphs in retinal axons, we generated snpha and snphb mutants using TALENmediated genome editing (Fig. $6 A, B$ ). We targeted a region upstream of the MTB to ensure full loss of function and obtained two alleles consisting of a 14 and 4 bp deletion for snpha and snphb, respectively, which were predicted to cause premature termination of translation (Fig. 6B). As both snpha and sphnb are expressed in RGCs (Fig. 5D) and to avoid potential compensation mechanisms, we generated maternal zygotic double mutants $(d b)$ and confirmed by RT-PCR and cDNA sequencing that both mutant mRNAs were expressed and contained the corresponding mutations (Fig. 6B). No alternative transcripts were detected in $d b$ (data not shown). $d b$ were viable and fertile, and $d b$ embryos did not show any obvious morphologic abnormalities, as was reported for Snph knock-out (KO) mice (Kang et al., 2008).
To test whether zebrafish Snphs also contribute to mitochondrial anchoring in axons, we quantified mitochondrial transport in mature retinal axons of WT and $d b$ embryos at $120 \mathrm{hpf}$ (Fig. 6C), when most mitochondria are known to become immobile (Lewis et al., 2016; Smit-Rigter et al., 2016). As expected, mitochondrial mobility was strongly increased in axons of $d b$ (Fig. $6 D, E$ ), demonstrating that the mitochondrial docking function of Snph is conserved in zebrafish.

We next performed time-lapse imaging of mitochondria in retinal axons elongating along the optic tract of $d b$ embryos to test whether Snphs also play a role in mitochondrial tethering at the growth cone of growing axons (Movies 6, 7, 8). We compared $d b$ with WT embryos that have a similar genetic background (datasets analyzed in Figs. 2-4). Interestingly, mitochondrial distribution at the growth cone was modified in $d b$ compared with WT (Fig. $7 A-D)$. While a same mitochondrial volume and number were observed in the growth cone peripheral volume and 
A
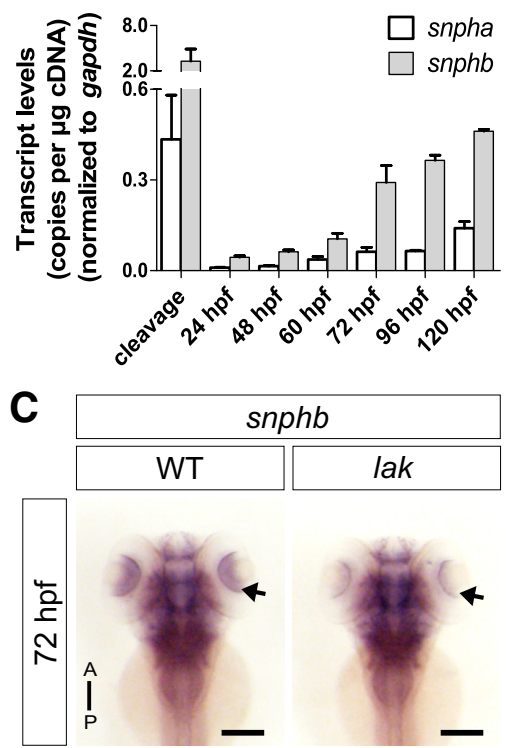

D

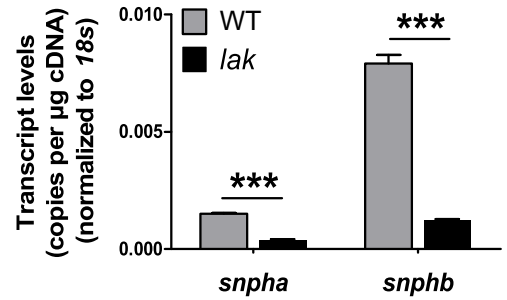

B

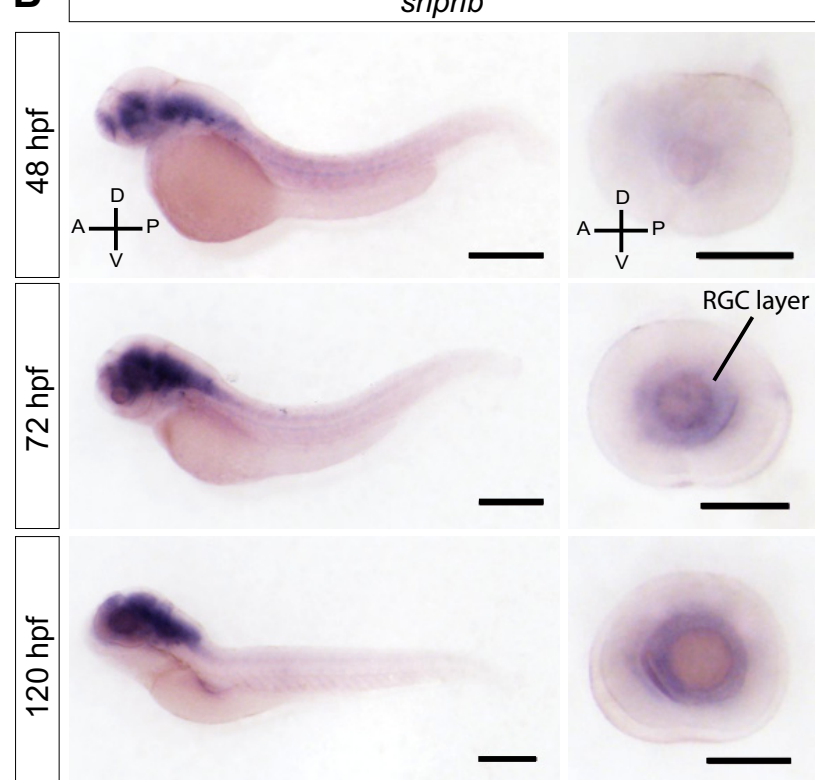

E
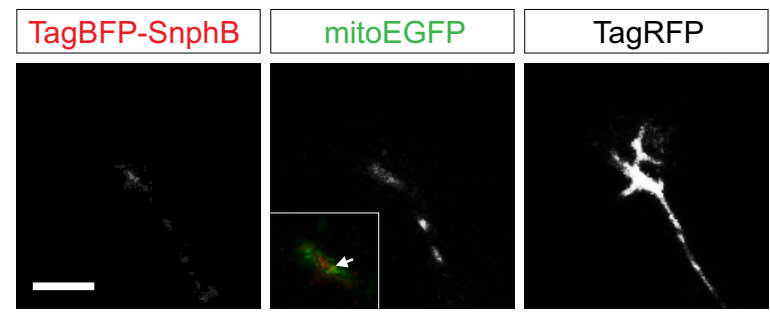

Figure 5. Zebrafish Syntaphilins are expressed in RGCs during development. A, Quantification of snpha and snphb mRNA levels during embryonic development by RT-ddPCR. mRNA levels were normalized to that of gapdh used as a control. Data from three independent experiments are shown as the mean \pm SEM. B, Lateral views of whole embryos stained for snphb by ISH show predominant expression in the brain at 48,72, and $120 \mathrm{hpf}$. snphb is also increasingly expressed in the RGC layer over time. Scale bars: whole embryos, $400 \mu \mathrm{m}$; eyes, $200 \mu \mathrm{m}$. C, Dorsal views of WT and RGC-deficient lak mutant embryos stained for snphb by ISH at $72 \mathrm{hpf}$. Expression of snphb is decreased in the retinae of lak embryos (arrows). Scale bar, $200 \mu \mathrm{m}$. D, Quantification of snpha and snphb mRNA levels in the eyes of WT and lak embryos at $72 \mathrm{hpf}$ analyzed by RT-ddPCR. Transcript levels were normalized to that of $18 \mathrm{~s}$, which was used as a control. Data from three experiments are shown as the mean \pm SEM. Statistical analysis: unpaired $t$ test, $* * * p<0.001$. E, TagBFP-Snphb localizes to the growth cone of elongating axons in vivo. Isl2b:TagBFP-snphb, isl2b:mitoEGFP2A-TagRFPCAAX, and isl2b:Lifeact-TagRFP were coexpressed in individual RGCs. TagBFP-Snphb and mitochondria are both present in the growth cone (arrow in merged image). Lateral view, confocal maximal projections. Scale bar, $5 \mu \mathrm{m}$.

area in WT and $d b$ (Fig. 7C,C), mitochondria occupied a smaller proportion of the growth cone total and central volumes and areas in $d b$ (Fig. $7 A, B^{\prime}$, Movies $6,7,8$ ). Moreover, mitochondria were located further from the growth cone leading edge in $d b$, which was most pronounced in pausing growth cones (Fig. $7 D, D)$ ). Interestingly, the decreased mitochondrial occupancy of the growth cone in $d b$ was accompanied with changes in mitochondrial flux. While a similar number of mitochondria arrived at the growth cone, more mitochondria left the growth cone in $d b$ versus WT (Fig. 7E), suggesting that Snphs dock mitochondria once they have reached the growth cone. The increased departure of mitochondria from the growth cone of $d b$ was associated with changes in net mitochondrial transport proximally to the growth cone. More mitochondria moved retrogradely in snph $d b$, which was accompanied by a decreased percentage of mitochondria moving in the anterograde direction (Fig. 7F). No differences were found between WT and $d b$ in the amount of time that mitochondria moved in both directions (Fig. 7G).

Finally, in addition to the decreased mitochondrial occupancy of the growth cone in $d b$, we observed some changes in pioneering mitochondrial clusters. As in WT, we detected pioneering clusters in every $d b$ growth cone that advanced (Fig. $8 A-C$ ). The number of clusters (Fig. $8 E$ ) and the amount of time they were present at the growth cone (Fig. 8F) did not vary; however, the total area occupied by pioneering mitochondrial clusters was significantly decreased in $d b$ compared with WT (Fig. 8D). Altogether, these observations demonstrate for the first time that Snphs contribute to mitochondrial clustering at the growth cone in vivo. Snphs play a role in anchoring mitochondria in the growth cone central domain, including both the main cluster and the pioneering mitochondria that dynamically appear along the leading edge of advancing growth cones. 
A

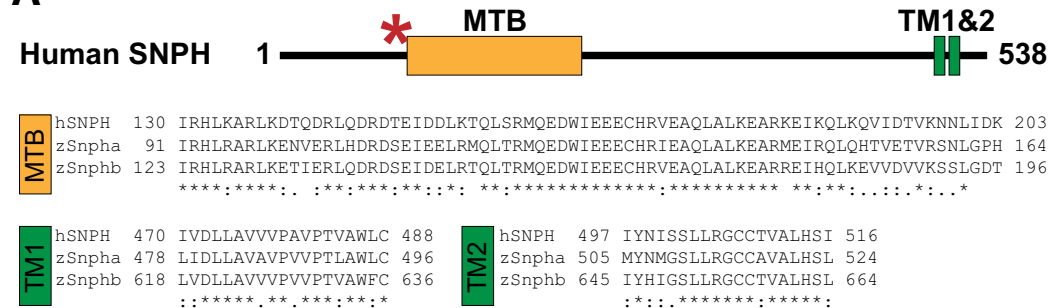

B

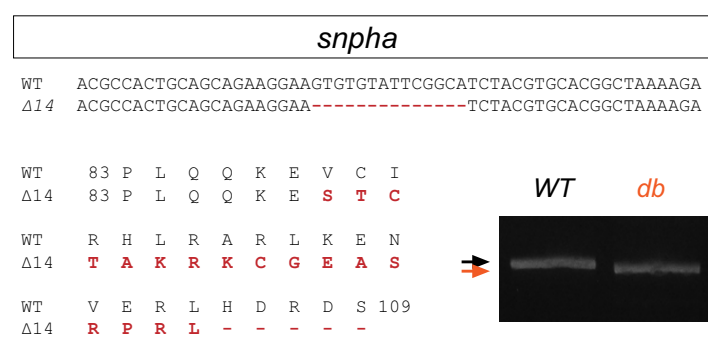

\section{snphb}

WT CTCCTGAGCAATACCTCACCCCACTGCAACAGAAAGAGGTGTGCATCAGGCACCTG $\triangle 4$ CTCCTGAGCAATACCTCACCCCACTG----AGAAAGAGGTGTGCATCAGGCACCTG

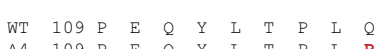

$\begin{array}{llllll}Y & L & T & P & L & R\end{array}$

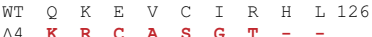

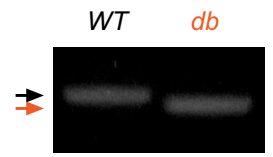

D
C

Mitochondrial transport in mature retinal axons

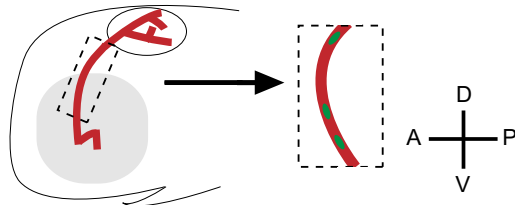

$120 \mathrm{hpf}$

Live imaging

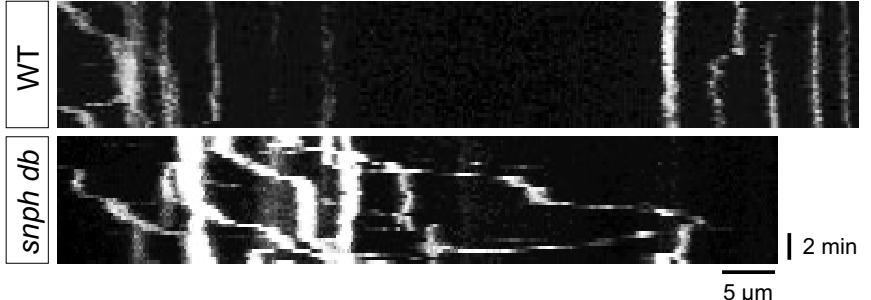

E

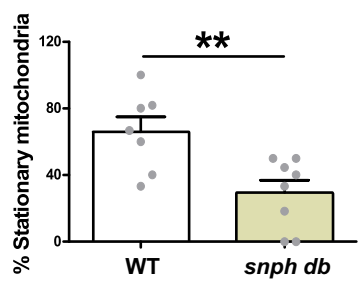

Figure 6. Zebrafish Syntaphilins anchor mitochondria in mature retinal axons. A, Domain structure of human SNPH. Both the MTB and TMs are highly conserved in zebrafish Snpha and Snphb (see also Extended Data Figure 6-1). The red asterisk indicates the position of the TALEN target region. $\boldsymbol{B}$, Mutations in snpha and snphb were introduced by TALEN mutagenesis. Red lines indicate the deleted sequences. Changes in amino acids are shown in red. RT-PCR analysis of snpha and snphb in WT and db embryos demonstrate the presence of shorter transcripts in the mutants. $\boldsymbol{C}$, Individual retinal axons and mitochondria were mosaically labeled by injecting isl2b:mitoEGFP-2A-TagRFPCAAX at the one-cell stage. After removal of the contralateral eye, mature axons of the optic tract and their mitochondria were imaged in a lateral view at $120 \mathrm{hpf}(\Delta t=15 \mathrm{~s})$. $\boldsymbol{D}, \boldsymbol{E}$, Quantification of the percentage of stationary mitochondria using kymograph analysis shows a reduction in stalled mitochondria in axons from snph db mutants. Data from two independent experiments (WT: $n=7, d b: n=8$ ) are shown as the mean \pm SEM. Statistical analysis: unpaired $t$ test, $* * p<0.01$.

\section{Mitochondrial docking at the growth cone by} Syntaphilins is not required for axon outgrowth

As Snphs contribute to mitochondrial docking at the growth cone (Figs. $7 A-G, 8)$, we then tested whether the

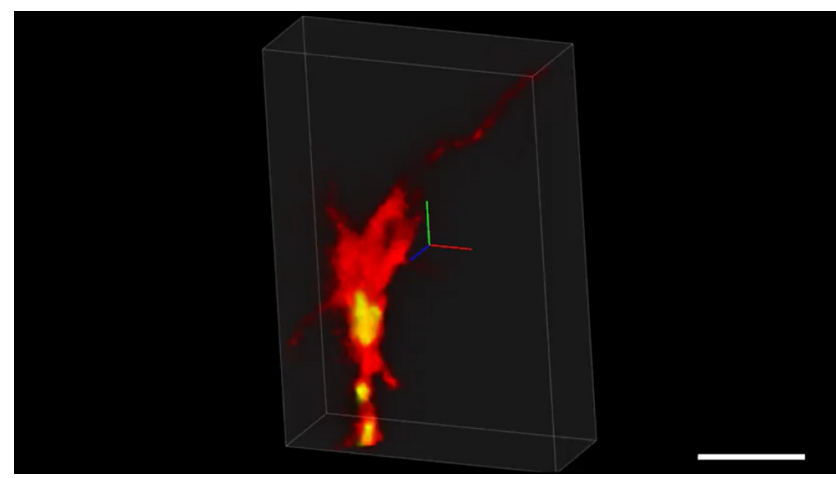

Movie 6. Representative 3D visualization of mitochondria in a pausing growth cone of a snph db mutant embryo. Video showing mitochondria (green) in a distal retinal axon and growth cone (red) pausing along the optic tract. Scale bar, 5 $\mu \mathrm{m}$. [View online]

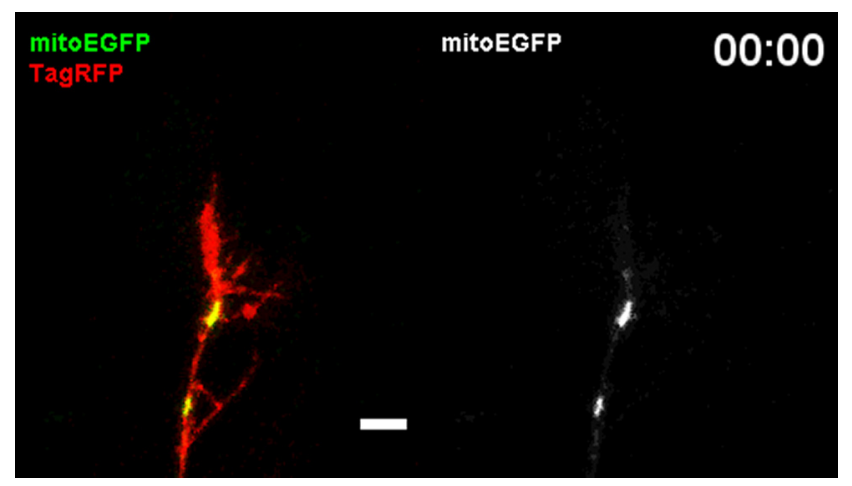

Movie 7. Representative time-lapse recording of mitochondrial dynamics in a growth cone of a snph $d b$ mutant embryo. Timelapse video showing mitochondria (green, white) in a distal retinal axon and growth cone (red) elongating along the optic tract of a snph $d b$ embryo. Images were acquired at 1 min intervals for 96 min. Note that the growth cone is combining periods of pausing with advancing (from 00:00 to 00:10 and 00:53 till end). Each frame is a confocal image stack maximal projection, lateral view, and anterior is on the left. Time stamp format: hours:minutes (hr:min). Scale bar, $5 \mu \mathrm{m}$. [View online] 


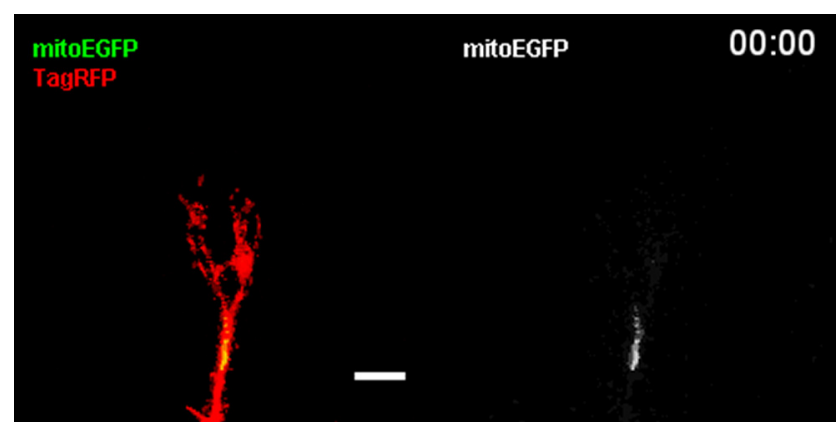

Movie 8. Representative time-lapse recording of mitochondrial dynamics in a growth cone of a snph $d b$ mutant embryo. Timelapse video showing mitochondria (green, white) in a distal retinal axon and the growth cone (red) elongating along the optic tract of a snph $d b$ embryo. Images were acquired at 1 min intervals for $120 \mathrm{~min}$. Note that the growth cone is pausing during the first minutes (00:00 to 00:47), after which it advances. Each frame is a confocal image stack maximal projection, lateral view, and anterior is on the left. Time stamp format: hours:minutes (hr:min). Scale bar, $5 \mu \mathrm{m}$. [View online]

loss of Snphs would affect axon elongation. Analysis of the total and central growth cone areas (Fig. $7 H$ ) and the number of filopodia (Fig. 7I) did not reveal any differences in growth cone morphology between WT and snph $d b$. Moreover, retinal axon growth rate was similar between WT and $d b$ mutants (Fig. 7J), indicating that Snphmediated mitochondrial docking at the growth cone is dispensable for axon elongation. Thus, we identified Snph as a mediator of mitochondrial docking at the growth cone in elongating axons in vivo, but this function plays only a minor role, if any, in axon growth.

\section{Discussion}

By performing confocal live imaging in the zebrafish embryo, we provide the first detailed characterization of mitochondrial dynamics during growth cone behavior in elongating axons in vivo. We show that mitochondrial distribution at and trafficking toward the growth cone are coordinated with axon outgrowth, which is in agreement with previous in vitro observations (Morris and Hollenbeck, 1993; Sainath et al., 2017) and highlights that mitochondria might play a role in axon outgrowth and pathfinding. We further provide evidence that Snph contributes to mitochondrial docking at the growth cone. However, growth cone morphology and axon elongation are unaffected in snph $\mathrm{db}$ mutants, indicating that the direct anchoring of mitochondria to growth cone microtubules by Snph plays only a minor role in axon elongation.

An intriguing finding of our study is the localization of mitochondria along growth cone filopodia that is independent of Snph. Since filopodia dynamics are highly dependent on actin filament polymerization and turnover, this observation suggests that mitochondria might associate with the growth cone actin cytoskeleton. Mitochondria have indeed been shown to be able to attach to actin via the myosin 19 molecular motor (Shneyer et al., 2016). Interaction with myosin 19 positioned mitochondria into actin-rich filopodia of U-2 OS osteosarcoma cells follow- ing stress (Shneyer et al., 2017). A localization of mitochondria to leading edge lamellipodia, filopodia, and invadopodia of cancer cells has further been shown to play an important role in cancer cell migration and metastatic potential (Cunniff et al., 2016; Smith and Gallo, 2018). Whether the interaction among mitochondria, myosin 19, and actin is relevant in the growth cone remains unknown. Interestingly, mitochondria appeared along growth cone filopodia in a transient manner, suggesting that they might also be transported along the dynamic microtubules that explore the growth cone periphery and play an essential role in growth cone adhesion and turning (Buck and Zheng, 2002; Suter et al., 2004). Our observation of pioneering mitochondrial clusters at the leading edge of the growth cone during elongation further supports the hypothesis that mitochondria might frequently attach to the plus-end of microtubules. An intriguing consequence of mitochondrial targeting to microtubule plus ends in filopodia would be the possibility to predict the net direction of axon growth based on mitochondrial appearance. Unfortunately, our imaging conditions did not have the resolution required for correlating mitochondrial peripheral distribution with filopodia protrusion, retraction, or stabilization. It will be important in the future to optimize the imaging approaches to quantify fine aspects of mitochondrial behavior in vivo and test the possible interaction between mitochondria and the cytoskeleton in the growth cone. Interestingly, the presence of pioneering mitochondrial clusters close to the leading edge is at least partially Snph dependent, suggesting that the targeting of mitochondria to peripheral filopodia and to the leading edge rely on different mechanisms. While mitochondria might be transported on actin filaments or dynamic microtubules in the periphery, they may accumulate on the stable microtubules that "end" in the central area of the growth cone and be captured there by Snph and other factors.

The interdependence between mitochondrial transport and axon outgrowth that we observed is consistent with in vitro work demonstrating a net anterograde mitochondrial trafficking in growing axons that is abolished when axons encounter a physical barrier (Morris and Hollenbeck, 1993). We now establish such a correlation for the first time in vivo by comparing various parameters of mitochondrial transport in pausing versus advancing retinal axons. Together with the observation of a growth statusdependent mitochondrial distribution at the growth cone, our data indicate that mitochondrial dynamics in the distal axon and growth cone are coordinated with growth cone behavior and axon outgrowth in vivo. Vaarmann et al. (2016) previously showed that axon length could be increased by overexpressing PGC- $1 \alpha$, a central inducer of mitochondrial biogenesis, supporting the notion that mitochondria provide energy for axon growth. In line with this, pharmacological and genetic disturbance of the mitochondrial fission-fusion balance in cultured retinal neurons affected neurite length and caused neurite guidance errors (Steketee et al., 2012). On the other hand, other studies hinted at the ability of axons to grow with dysfunctional mitochondria (Yoon et al., 2012; Campbell et al., 2014). While depletion of the intermediate filament 


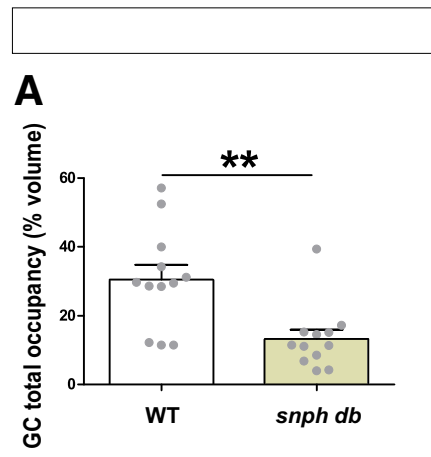

Mitochondrial distribution
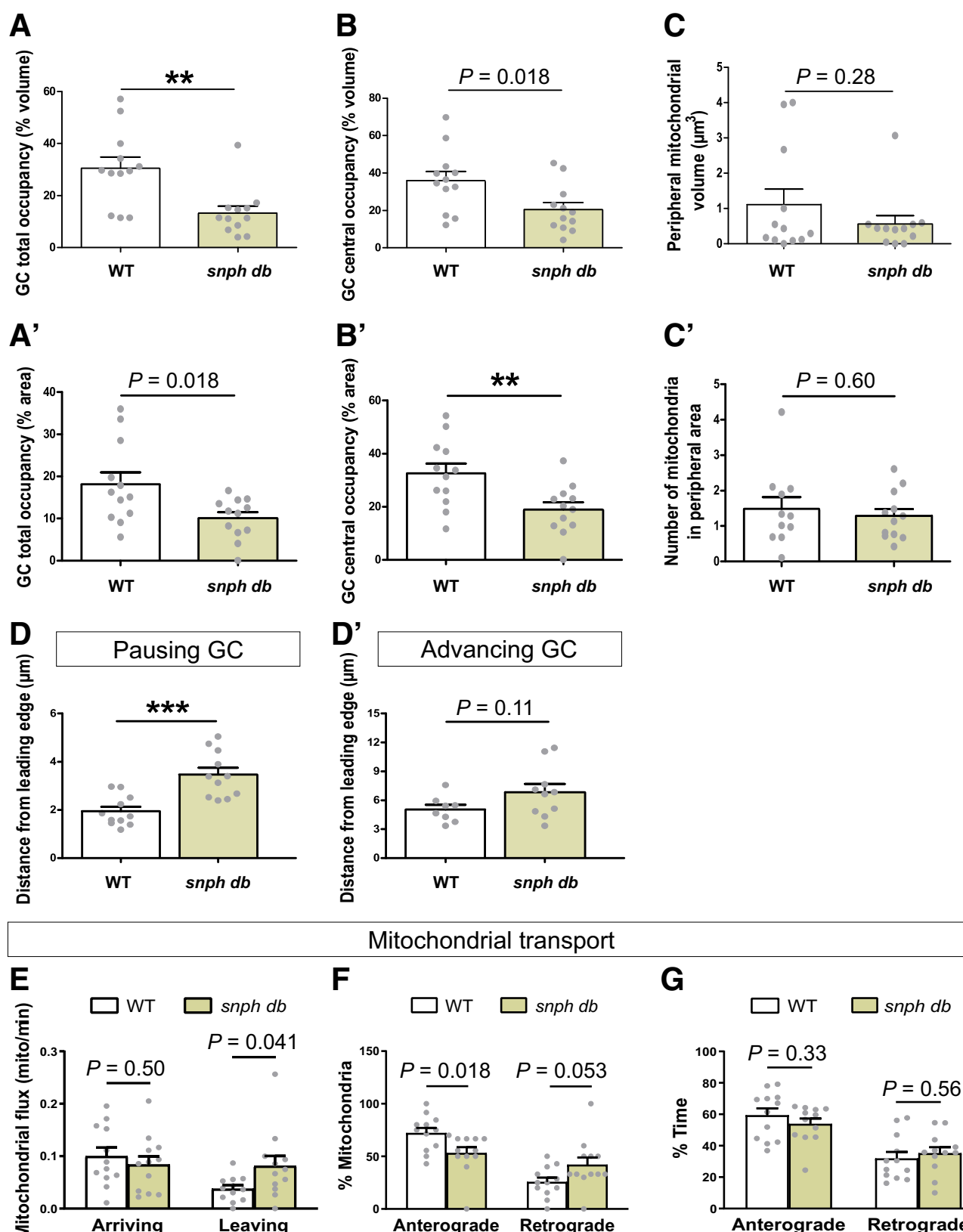

\section{Mitochondrial transport}

$\mathbf{F}$
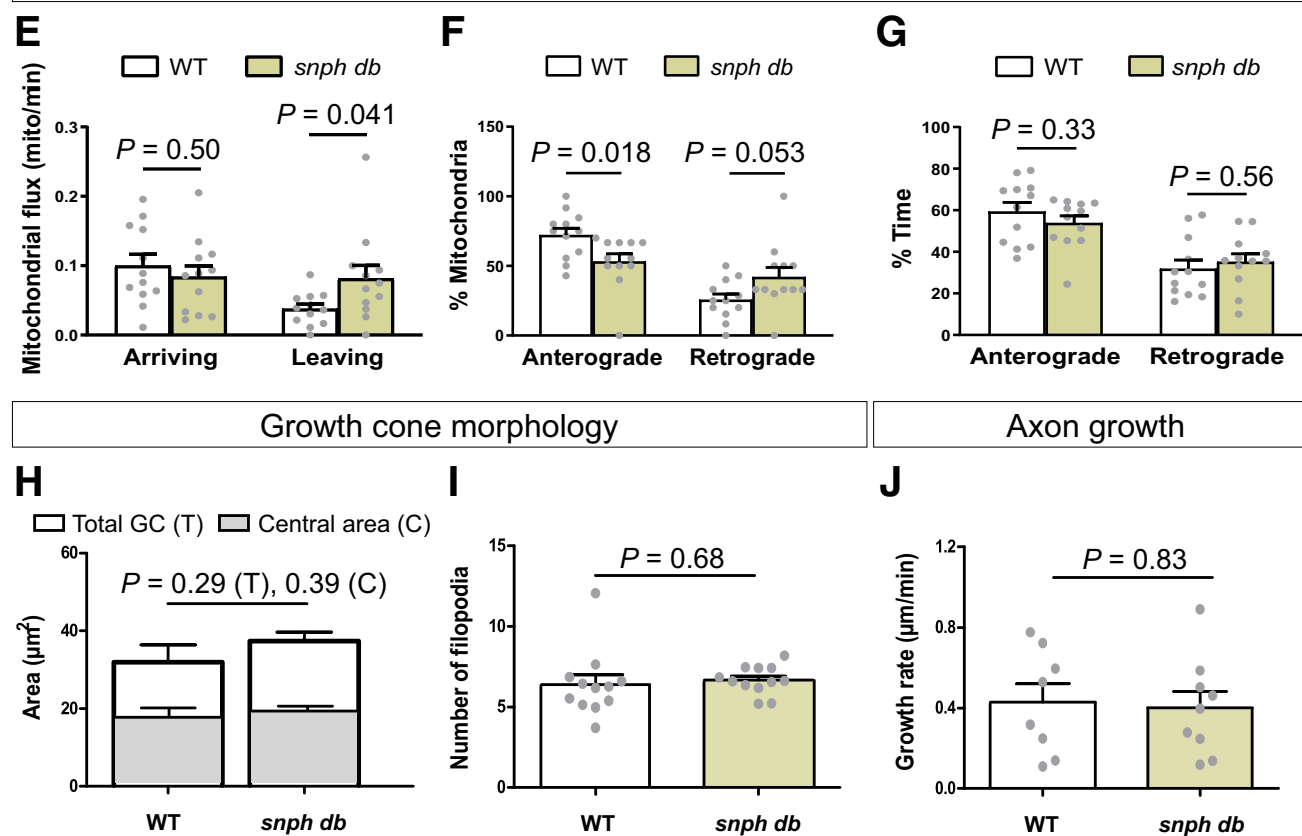

Figure 7. Syntaphilins participate in mitochondrial docking at the growth cone but do not regulate axon elongation. $\boldsymbol{A}-\boldsymbol{B}^{\prime}$, Quantification of the mitochondrial occupancy (as a percentage) of the growth cone total volume $(\boldsymbol{A})$, total area $(\dot{\boldsymbol{A}})$, central volume $(\boldsymbol{B})$, and central area (B) in WT and snph db. Mitochondrial occupancy is decreased in snph db. C, $\boldsymbol{C}^{\prime}$, Quantification of the mitochondrial 
continued

volume in the peripheral growth cone $(\boldsymbol{C})$, and of the number of mitochondria in the growth cone peripheral area (C) in WT and snph db. $\boldsymbol{D}, \boldsymbol{D}^{\prime}$, Quantification of the distance between the largest mitochondrial cluster and the growth cone leading edge in WT and snph $d b$. We quantified the distance from the leading edge separately in pausing and advancing growth cones as it varies depending on growth cone status (Fig. 2E). Mitochondria are located further from the leading edge in retinal growth cones of $d b$ compared with WT. $\boldsymbol{E}-\boldsymbol{G}$, Reduced mitochondrial occupancy in the growth cone of snph $d b$ is accompanied by an increased removal of mitochondria from the growth cone. More mitochondria leave the growth cone per minute in $d b$, while no difference is detected for arriving mitochondria $(\boldsymbol{E})$. Quantification of net mitochondrial transport proximally to the growth cone $(\boldsymbol{F})$ shows more mitochondria moving retrogradely in snph $d b$, which is accompanied by a decreased percentage of mitochondria moving in the anterograde direction. No differences in percentage time in anterograde or retrograde motion were found $(\boldsymbol{G})$. $\boldsymbol{H}, \boldsymbol{I}$, Quantification of growth cone morphology shows no differences in growth cone total and central areas $(\boldsymbol{H})$ and number of filopodia $(\boldsymbol{I})$ between $d b$ and WT embryos. $\boldsymbol{J}$, Axon elongation is not statistically different between $d b$ and WT embryos. Data from 12 independent experiments per genotype are shown as the mean \pm SEM. Statistical analysis: unpaired $t$ test, $* * p<0.01, * * * p<0.001$

protein Lamin B2 caused defects in mitochondrial membrane potential, morphology, and transport, it did not affect retinal axon initial growth and guidance along the optic tract in vivo (Yoon et al., 2012). In a similar manner, lack of Kif5Aa, which transports mitochondria anterogradely into axons, causes a lack of mitochondria in distal peripheral axons that leads to degeneration. Yet, axons grow normally in kif5Aa zebrafish mutant embryos (Campbell et al., 2014). As it remains unclear which cellular processes are specifically dependent on mitochondrial respiration (Smith and Gallo, 2018), it might be conceivable that ATP production through glycolysis can, at least partially, sustain axon growth. Previous studies have even suggested that embryonic neurons predominantly rely on

A
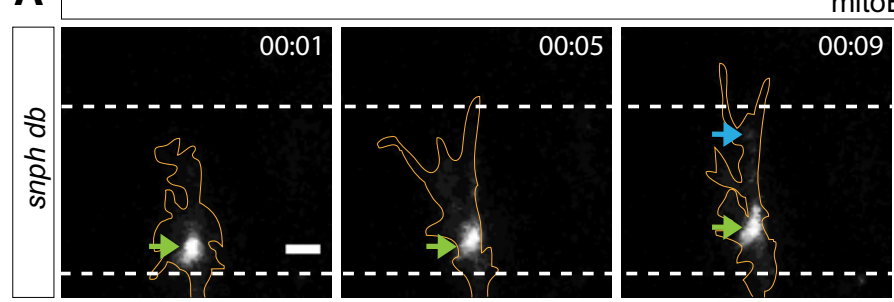

mitoEGFP
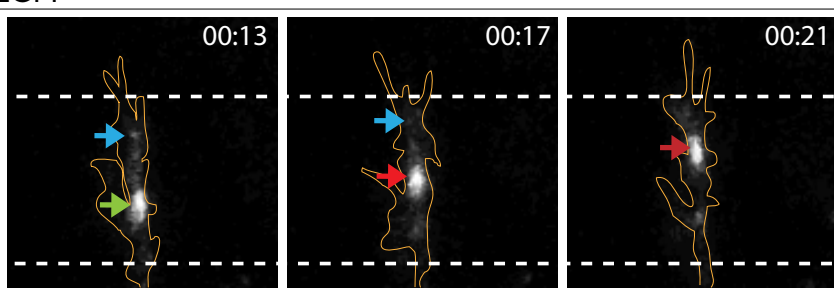

B
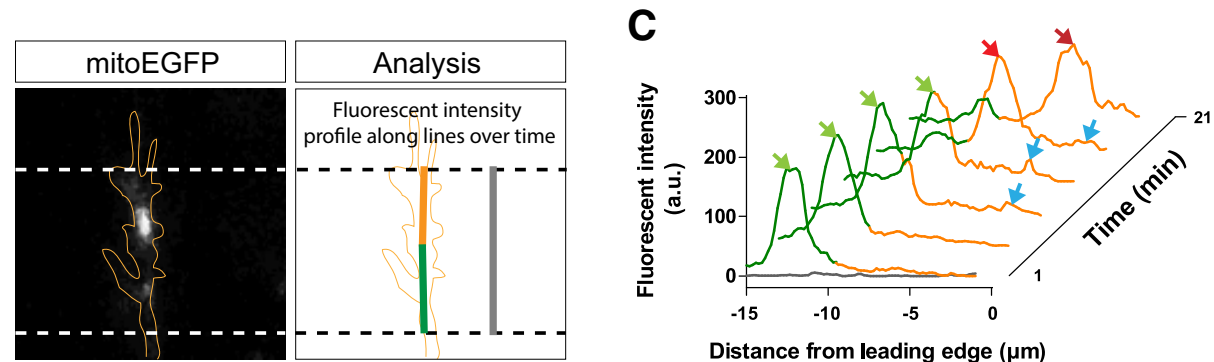

Distance from leading edge $(\mu \mathrm{m})$

D

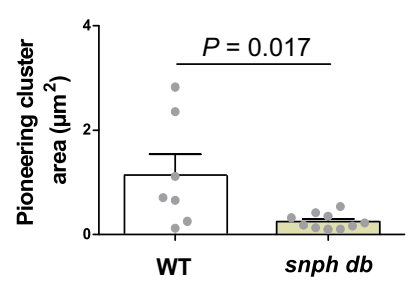

E

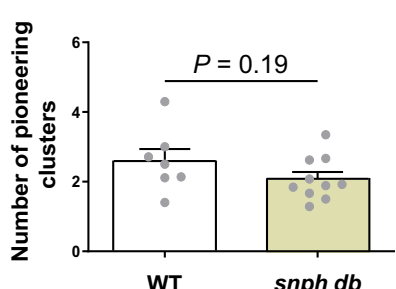

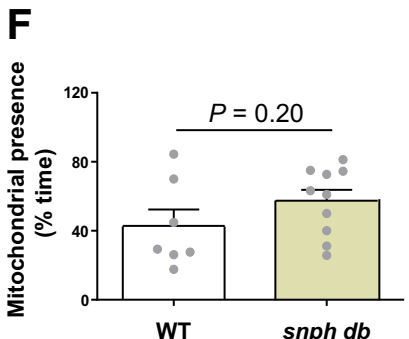

Figure 8. Syntaphilin contributes to the localization of pioneering mitochondrial clusters near the leading edge in advancing growth cones. $\boldsymbol{A}$, Representative time-lapse images of mitochondria (mitoEGFP, white) in an advancing growth cone (delineated in orange) in a $d b$ embryo. Like in WT (Fig. 3), the main mitochondrial cluster lags behind during growth cone advance while some pioneering mitochondria appear in close proximity to the leading edge. Lateral view, Confocal maximal projections. Scale bar, $3 \mu \mathrm{m}$. $\boldsymbol{B}$, C, Fluorescent intensity profiles show the distribution of mitoEGFP fluorescence at various time points along the advancing growth cone shown in $\boldsymbol{A}$. Orange and green lines correspond to fluorescent intensities of mitochondria in the growth cone and the proximal growth cone, respectively. Arrows show peaks of fluorescence corresponding to localizations of mitochondria in the growth cone (see $\boldsymbol{A}$ ). $\boldsymbol{D}-\boldsymbol{F}$, Quantification of pioneering mitochondrial cluster total area $(\boldsymbol{D})$, number $(\boldsymbol{E})$, and dynamics (percentage of time present during advance; $\boldsymbol{F}$ ). Pioneering mitochondrial clusters occupy a reduced area in snph $d b$ embryos compared with WT. Data from 12 independent experiments per genotype are shown as the mean \pm SEM. Statistical analysis: unpaired $t$ test. 
glycolytic ATP (Surin et al., 2012). As growth cone turning is regulated by local calcium levels (Gasperini et al., 2017), the ability of growth cone mitochondria to buffer calcium might be more important than its capacity to generate energy. Future work analyzing mitochondrial calcium dynamics at the growth cone in vivo might thus be highly interesting.

We identified Snph as a mediator of mitochondrial docking at the growth cone in elongating axons in vivo. This function is consistent with its previously established role in axonal mitochondrial stalling (Kang et al., 2008; Chen and Sheng, 2013). Yet, our data suggest that Snph does not play an important role in stalling mitochondria along the axon at this early developmental stage. The lack of difference in the number of mitochondria arriving at the growth cone in WT versus snph $d b$ axons suggests that Snph does not inhibit mitochondrial anterograde transport toward the growth cone as it does in the context of axon regeneration (Zhou et al., 2016). An increased halting of mitochondria along the axon over time is instead consistent with an increased expression of Snph in the brain during development, both in zebrafish in our study and in the mouse (Das et al., 2003; Kang et al., 2008). Alternatively, Snph localization and functions might be differently regulated in the axon shaft versus growth cone during development.

While the loss of Snphs reduced the number of mitochondria at the growth cone, it did not cause a complete depletion of mitochondria and did not affect axon growth. The remaining mitochondria at the growth cone might thus compensate for a lower density by increasing their activity. In this regard, it is noteworthy that Snph KO mice sciatic nerves show enhanced regrowth capacity after injury (Zhou et al., 2016). Cultured cortical neurons from these Snph KO mice displayed increased ATP content on axotomy (Zhou et al., 2016), but this difference in energy production was not observed in baseline conditions. Alternatively, the number of mitochondria remaining at the growth cone in the absence of Snph might be enough for normal axon development, especially considering that glycolytic ATP might have an important role in sustaining axon growth (Surin et al., 2012). These remaining mitochondria are likely stabilized at the growth cone central domain by other anchoring factors, of which the outer mitochondrial membrane GTPases Miro1 and 2 are especially good candidates. Miro proteins are indeed involved in mitochondrial trafficking along microtubules (Devine et al., 2016) and can arrest mitochondrial movement in a calcium-dependent manner (Saotome et al., 2008), which is particularly relevant at the growth cone where calcium signaling regulates pausing, extension, and turning (Sutherland et al., 2014; Gasperini et al., 2017). In that context, an elegant study recently demonstrated that growth inhibiting substrates such as MAG or chondroitin sulfate proteoglycans decrease mitochondrial axonal transport by promoting the deacetylation of Miro1 by HDAC6 in a calcium-dependent manner (Kalinski et al., 2019). Finally, Miros have also been discovered to play a role in mitochondrial trafficking and positioning along actin filaments (López-Doménech et al., 2018), which might regulate mitochondrial positioning along filopodia in the growth cone peripheral area. Future studies examining the role of Miros in mitochondrial docking at the growth cone in vivo will thus be of great interest to decipher how mitochondrial positioning is regulated during axonal development.

\section{References}

Beck H, Flynn K, Lindenberg KS, Schwarz H, Bradke F, Di Giovanni S, Knoll B (2012) Serum Response Factor (SRF)-cofilin-actin signaling axis modulates mitochondrial dynamics. Proc Natl Acad Sci U S A 109:E2523-E2532.

Bertholet AM, Delerue T, Millet AM, Moulis MF, David C, Daloyau M, Arnauné-Pelloquin L, Davezac N, Mils V, Miquel MC, Rojo M, Belenguer P (2016) Mitochondrial fusion/fission dynamics in neurodegeneration and neuronal plasticity. Neurobiol Dis 90:3-19.

Boldogh IR, Pon LA (2006) Interactions of mitochondria with the actin cytoskeleton. Biochim Biophys Acta 1763:450-462.

Bovolenta P, Mason C (1987) Growth cone morphology varies with position in the developing mouse visual pathway from retina to first targets. J Neurosci 7:1447-1460.

Buck KB, Zheng JQ (2002) Growth cone turning induced by direct local modification of microtubule dynamics. J Neurosci 22:93589367.

Campbell PD, Shen K, Sapio MR, Glenn TD, Talbot WS, Marlow FL (2014) Unique function of Kinesin Kif5A in localization of mitochondria in axons. J Neurosci 34:14717-14732.

Cermak T, Doyle EL, Christian M, Wang L, Zhang Y, Schmidt C, Baller JA, Somia NV, Bogdanove AJ, Voytas DF (2011) Efficient design and assembly of custom TALEN and other TAL effectorbased constructs for DNA targeting. Nucleic Acids Res 39:e82.

Chen Y, Sheng ZH (2013) Kinesin-1-syntaphilin coupling mediates activity-dependent regulation of axonal mitochondrial transport. J Cell Biol 202:351-364.

Courchet J, Lewis TL Jr, Lee S, Courchet V, Liou DY, Aizawa S, Polleux F (2013) Terminal axon branching is regulated by the LKB1-NUAK1 kinase pathway via presynaptic mitochondrial capture. Cell 153:1510-1525.

Cunniff B, McKenzie AJ, Heintz NH, Howe AK (2016) AMPK activity regulates trafficking of mitochondria to the leading edge during cell migration and matrix invasion. Mol Biol Cell 27:2662-2674.

Dahlem TJ, Hoshijima K, Jurynec MJ, Gunther D, Starker CG, Locke AS, Weis AM, Voytas DF, Grunwald DJ (2012) Simple methods for generating and detecting locus-specific mutations induced with TALENs in the zebrafish genome. PLoS Genet 8:e1002861.

Das S, Boczan J, Gerwin C, Zald PB, Sheng ZH (2003) Regional and developmental regulation of syntaphilin expression in the brain: a candidate molecular element of synaptic functional differentiation. Brain Res Mol Brain Res 116:38-49.

Devine MJ, Birsa N, Kittler JT (2016) Miro sculpts mitochondrial dynamics in neuronal health and disease. Neurobiol Dis 90:27-34.

Drerup CM, Herbert AL, Monk KR, Nechiporuk AV (2017) Regulation of mitochondria-dynactin interaction and mitochondrial retrograde transport in axons. Elife 6:e22234.

Gasperini RJ, Pavez M, Thompson AC, Mitchell CB, Hardy H, Young KM, Chilton JK, Foa L (2017) How does calcium interact with the cytoskeleton to regulate growth cone motility during axon pathfinding? Mol Cell Neurosci 84:29-35.

Gaynes JA, Otsuna H, Campbell DS, Manfredi JP, Levine EM, Chien CB (2015) The RNA binding protein Igf2bp1 is required for zebrafish RGC axon outgrowth in vivo. PLoS One 10:e0134751.

Glasauer SM, Neuhauss SC (2014) Whole-genome duplication in teleost fishes and its evolutionary consequences. Mol Genet Genomics 289:1045-1060.

Holt CE, Harris WA (1993) Position, guidance, and mapping in the developing visual system. J Neurobiol 24:1400-1422.

Kalinski AL, Kar AN, Craver J, Tosolini AP, Sleigh JN, Lee SJ, Hawthorne A, Brito-Vargas P, Miller-Randolph S, Passino R, Shi L, 
Wong VSC, Picci C, Smith DS, Willis DE, Havton LA, Schiavo G, Giger RJ, Langley B, Twiss JL (2019) Deacetylation of Miro1 by HDAC6 blocks mitochondrial transport and mediates axon growth inhibition. J Cell Biol 218:1871-1890.

Kang JS, Tian JH, Pan PY, Zald P, Li C, Deng C, Sheng ZH (2008) Docking of axonal mitochondria by syntaphilin controls their mobility and affects short-term facilitation. Cell 132:137-148.

Kay JN, Finger-Baier KC, Roeser T, Staub W, Baier H (2001) Retinal ganglion cell genesis requires lakritz, a Zebrafish atonal homolog. Neuron 30:725-736.

Kimmel CB, Ballard WW, Kimmel SR, Ullmann B, Schilling TF (1995) Stages of embryonic development of the zebrafish. Dev Dyn 203: 253-310.

Kwan KM, Fujimoto E, Grabher C, Mangum BD, Hardy ME, Campbell DS, Parant JM, Yost HJ, Kanki JP, Chien CB (2007) The Tol2kit: a multisite gateway-based construction kit for Tol2 transposon transgenesis constructs. Dev Dyn 236:3088-3099.

Lewis TL Jr, Turi GF, Kwon SK, Losonczy A, Polleux F (2016) Progressive decrease of mitochondrial motility during maturation of cortical axons in vitro and in vivo. Curr Biol 26:2602-2608.

Liew WC, Bartfai R, Lim Z, Sreenivasan R, Siegfried KR, Orban L (2012) Polygenic sex determination system in zebrafish. PLoS One 7:e34397.

López-Doménech G, Covill-Cooke C, Ivankovic D, Halff EF, Sheehan DF, Norkett R, Birsa N, Kittler JT (2018) Miro proteins coordinate microtubule- and actin-dependent mitochondrial transport and distribution. EMBO J 37:321-336.

Maack G, Segner H (2003) Morphological development of the gonads in zebrafish. J Fish Biol 62:895-906.

Mason CA, Wang LC (1997) Growth cone form is behavior-specific and, consequently, position-specific along the retinal axon pathway. J Neurosci 17:1086-1100.

Melkov A, Abdu U (2018) Regulation of long-distance transport of mitochondria along microtubules. Cell Mol Life Sci 75:163-176.

Misko A, Jiang S, Wegorzewska I, Milbrandt J, Baloh RH (2010) Mitofusin 2 is necessary for transport of axonal mitochondria and interacts with the Miro/Milton complex. J Neurosci 30:4232-4240.

Moriyoshi K, Richards LJ, Akazawa C, O'Leary DD, Nakanishi S (1996) Labeling neural cells using adenoviral gene transfer of membrane-targeted GFP. Neuron 16:255-260.

Morris RL, Hollenbeck PJ (1993) The regulation of bidirectional mitochondrial transport is coordinated with axonal outgrowth. J Cell Sci 104:917-927.

Neumann S, Chassefeyre R, Campbell GE, Encalada SE (2017) KymoAnalyzer: a software tool for the quantitative analysis of intracellular transport in neurons. Traffic 18:71-88.

Ota S, Hisano Y, Muraki M, Hoshijima K, Dahlem TJ, Grunwald DJ, Okada Y, Kawahara A (2013) Efficient identification of TALENmediated genome modifications using heteroduplex mobility assays. Genes Cells 18:450-458.

Parant JM, George SA, Pryor R, Wittwer CT, Yost HJ (2009) A rapid and efficient method of genotyping zebrafish mutants. Dev Dyn 238:3168-3174.

Pittman AJ, Law MY, Chien CB (2008) Pathfinding in a large vertebrate axon tract: isotypic interactions guide retinotectal axons at multiple choice points. Development 135:2865-2871.

Plucińska G, Paquet D, Hruscha A, Godinho L, Haass C, Schmid B, Misgeld T (2012) In vivo imaging of disease-related mitochondrial dynamics in a vertebrate model system. J Neurosci 32:1620316212.

Poulain FE, Chien CB (2013) Proteoglycan-mediated axon degeneration corrects pretarget topographic sorting errors. Neuron 78:4956.

Poulain FE, Gaynes JA, Stacher Hörndli C, Law MY, Chien CB (2010) Analyzing retinal axon guidance in zebrafish. Methods Cell Biol 100:3-26.

Provost E, Rhee J, Leach SD (2007) Viral 2A peptides allow expression of multiple proteins from a single ORF in transgenic zebrafish embryos. Genesis 45:625-629.
Riedl J, Crevenna AH, Kessenbrock K, Yu JH, Neukirchen D, Bista M, Bradke F, Jenne D, Holak TA, Werb Z, Sixt M, Wedlich-Soldner R (2008) Lifeact: a versatile marker to visualize F-actin. Nat Methods 5:605-607.

Sainath R, Armijo-Weingart L, Ketscheck A, Xu Z, Li S, Gallo G (2017) Chondroitin sulfate proteoglycans negatively regulate the positioning of mitochondria and endoplasmic reticulum to distal axons. Dev Neurobiol 77:1351-1370.

Sakuma T, Hosoi S, Woltjen K, Suzuki K, Kashiwagi K, Wada H, Ochiai H, Miyamoto T, Kawai N, Sasakura Y, Matsuura S, Okada Y, Kawahara A, Hayashi S, Yamamoto T (2013) Efficient TALEN construction and evaluation methods for human cell and animal applications. Genes Cells 18:315-326.

Saotome M, Safiulina D, Szabadkai G, Das S, Fransson A, Aspenstrom P, Rizzuto R, Hajnóczky G (2008) Bidirectional Ca2+dependent control of mitochondrial dynamics by the Miro GTPase. Proc Natl Acad Sci U S A 105:20728-20733.

Schindelin J, Arganda-Carreras I, Frise E, Kaynig V, Longair M, Pietzsch T, Preibisch S, Rueden C, Saalfeld S, Schmid B, Tinevez JY, White DJ, Hartenstein V, Eliceiri K, Tomancak P, Cardona A (2012) Fiji: an open-source platform for biological-image analysis. Nat Methods 9:676-682.

Schneider CA, Rasband WS, Eliceiri KW (2012) NIH Image to ImageJ: 25 years of image analysis. Nat Methods 9:671-675.

Shneyer BI, Ušaj M, Henn A (2016) Myo19 is an outer mitochondrial membrane motor and effector of starvation-induced filopodia. $\mathrm{J}$ Cell Sci 129:543-556.

Shneyer BI, Ušaj M, Wiesel-Motiuk N, Regev R, Henn A (2017) ROS induced distribution of mitochondria to filopodia by Myo19 depends on a class specific tryptophan in the motor domain. Sci Rep 7:11577.

Smit-Rigter L, Rajendran R, Silva CA, Spierenburg L, Groeneweg F, Ruimschotel EM, van Versendaal D, van der Togt C, Eysel UT, Heimel JA, Lohmann C, Levelt CN (2016) Mitochondrial dynamics in visual cortex are limited in vivo and not affected by axonal structural plasticity. Curr Biol 26:2609-2616.

Smith GM, Gallo G (2018) The role of mitochondria in axon development and regeneration. Dev Neurobiol 78:221-237.

Steketee MB, Moysidis SN, Weinstein JE, Kreymerman A, Silva JP, Iqbal S, Goldberg JL (2012) Mitochondrial dynamics regulate growth cone motility, guidance, and neurite growth rate in perinatal retinal ganglion cells in vitro. Invest Ophthalmol Vis Sci 53:74027411.

Surin AM, Khiroug S, Gorbacheva LR, Khodorov BI, Pinelis VG, Khiroug $L$ (2012) Comparative analysis of cytosolic and mitochondrial ATP synthesis in embryonic and postnatal hippocampal neuronal cultures. Front Mol Neurosci 5:102.

Suter DM, Schaefer AW, Forscher P (2004) Microtubule dynamics are necessary for SRC family kinase-dependent growth cone steering. Curr Biol 14:1194-1199.

Sutherland DJ, Pujic Z, Goodhill GJ (2014) Calcium signaling in axon guidance. Trends Neurosci 37:424-432.

Thévenaz P, Ruttimann UE, Unser M (1998) A pyramid approach to subpixel registration based on intensity. IEEE Trans Image Process 7:27-41.

Thisse C, Thisse B (2008) High-resolution in situ hybridization to whole-mount zebrafish embryos. Nat Protoc 3:59-69.

Trevisan T, Pendin D, Montagna A, Bova S, Ghelli AM, Daga A (2018) Manipulation of mitochondria dynamics reveals separate roles for form and function in mitochondria distribution. Cell Rep 23:17421753.

Vaarmann A, Mandel M, Zeb A, Wareski P, Liiv J, Kuum M, Antsov E, Liiv M, Cagalinec M, Choubey V, Kaasik A (2016) Mitochondrial biogenesis is required for axonal growth. Development 143:19811992.

Wan Y, Otsuna H, Holman HA, Bagley B, Ito M, Lewis AK, Colasanto M, Kardon G, Ito K, Hansen C (2017) FluoRender: joint freehand segmentation and visualization for many-channel fluorescence data analysis. BMC Bioinformatics 18:280. 
Wehnekamp F, Plucińska G, Thong R, Misgeld T, Lamb DC (2019) Nanoresolution real-time 3D orbital tracking for studying mitochondrial trafficking in vertebrate axons in vivo. Elife 8:e46059.

Yoon BC, Jung H, Dwivedy A, O'Hare CM, Zivraj KH, Holt CE (2012) Local translation of extranuclear lamin $\mathrm{B}$ promotes axon maintenance. Cell 148:752-764.
Zala D, Hinckelmann MV, Yu H, Lyra da Cunha MM, Liot G, Cordelières FP, Marco S, Saudou F (2013) Vesicular glycolysis provides on-board energy for fast axonal transport. Cell 152:479-491.

Zhou B, Yu P, Lin MY, Sun T, Chen Y, Sheng ZH (2016) Facilitation of axon regeneration by enhancing mitochondrial transport and rescuing energy deficits. J Cell Biol 214:103-119. 